\title{
Dispersion of Waves in a Cold Magnetoplasma From Hydromagnetic to Whistler Frequencies
}

\author{
Henry G. Booker ${ }^{1}$ and Rolf B. Dyce ${ }^{2}$
}

(Received May 19, 1964; revised November 30, 1964)

\begin{abstract}
It is shown that dispersion of waves in the magnetosphere regarded as a cold plasma may conveniently be handled from whistler frequencies down to frequencies small compared with the ionic gyrofrequency by an approximation (22) similar to the Appleton-Hartree approximation (23) used at radio frequencies. In both cases the ratio of the ionic mass to the electronic mass is assumed infinite, but whereas for the radio approximation this is achieved by taking the ionic mass infinite, it is achieved for the hydromagnetic approximation by taking the electronic mass zero. This permits extension of the concept of quasi-longitudinal and quasi-transverse approximations to the entire frequency spectrum (fig. 2); propagation is predominantly quasi-transverse at frequencies small compared with the ionic gyrofrequency. The directional pulse patterns for radiation of a pulse from a source are calculated (fig. 11) and the transition from the omnidirectional MHD wave to the whistler mode is illustrated (fig. 13). The hydromagnetic approximation here used facilitates detailed calculation of elliptical polarization (figs. 14-19) and space-charge (fig. 20). Attenuation due to ionic collisions with neutral gas is calculated (fig. 21) on the assumption that the neutral gas is too massive to take part in the wave motion.
\end{abstract}

\section{Introduction}

For many purposes the ionosphere and the magnetosphere may be regarded as a cold magnetoplasma. Considerable interest attaches to understanding the propagation of waves in such a plasma from frequencies small compared with the ionic gyrofrequency to frequencies large compared with the electronic plasma frequency. The radio frequency end of the spectrum has been studied for a number of decades and is understood in considerable detail. Reference may be made to books by Ratcliffe [1959] and by Budden [1961]. The hydromagnetic end of the spectrum was developed independently by Alfvén [1950], and has become an important aspect of fluid mechanics. It has long been realized, however, that, if both ions and electrons are taken into account, the phenomena at the two ends of the spectrum are both incorporated in the magnetoionic theory [Aström, 1950]. The unity of the theory at radio and hydromagnetic frequencies has been brought out in recent books by Denisse and Delcroix [1961], by Stix [1962], and by Allis, Buchsbaum, and Bers [1963]. However, the complete version of the magneto-ionic theory required to cover the entire spectrum is algebraically complicated. For radiation from a source in a cold uniform magnetoplasma, the various topological forms taken by the phase propagation surfaces have been ascertained and are conveniently demonstrated in a diagram of the type introduced by Clemmow and Mullaly [1955], and modified by Allis [1959]. However, by making appropriate approximations, one can concentrate on those situations of greatest interest in the ionosphere and the magnetosphere, and this permits a simpler treatment that facilitates greater understanding. The best known simplification of this type is, of course, the Appleton-Hartree formula, [Appleton, 1932; Hartree, 1931] together with its quasi-longitudinal and quasi-transverse approximations [Booker, 1935]. We shall describe the Appleton-Hartree formula as based on the radio approximation to the complete magneto-ionic theory. It is the objective of this paper to make corresponding use of a similar approximation covering the hydromagnetic end of the spectrum. Together, the hydromagnetic and radio approximations cover the vast majority of situations of practical interest in the magnetosphere regarded as a cold plasma.

In both the radio and the hydromagnetic approximations it is assumed that the ratio of the mass of an electron to the mass of an ion is negligibly small. In the radio approximation this is

${ }^{1}$ Cornell University, Ithaca, New York; work done while on leave of absence at the Stanford Research Institute.

${ }^{2}$ Stanford Research Institute, Menlo Park, Calif.; curręntly on leave of absence at Cornell University, Arecibo Ionospheric Observatory, Puerto Rico. 
achieved by allowing fully for the inertia of the electrons but taking the mass of an ion as infinite. In the hydromagnetic approximation, on the other hand, we allow fully for the inertia of the ions, but take the mass of an electron as zero. At whistler frequencies, under conditions of applicability of the Eckersley law [Eckersley, 1935], the two approximations give the same results, thus permitting a good fit between the hydromagnetic and radio approximations under conditions of practical interest.

The two elliptically polarized waves under discussion are known at radio frequencies as the ordinary and extraordinary waves ( $\mathrm{O}$ and $\mathrm{X}$ waves). The $\mathrm{O}$ wave is the wave for which propagation becomes independent of the imposed magnetic field when the direction of this field is turned so as to be perpendicular to the direction of phase propagation. ${ }^{3}$ Below the ionic gyrofrequency these waves may also be referred to as the slow and fast waves respectively. At frequencies small compared with the ionic gyrofrequency they are referred to in the fluid mechanics literature [Bershader, 1959] as the transverse and fast waves, and the term slow is reserved for the acoustic mode. The two waves may also be classified according to the direction of rotation of the electric vector round the direction of the imposed magnetic field. This is actually the most convenient classification within the confines of the hydromagnetic approximation used in this paper. In this approximation the assumption of zero electronic mass gives infinite conductivity in the direction of the imposed magnetic field. This makes the plane of the electric ellipse perpendicular to the imposed magnetic field for all directions of propagation. We shall describe waves as left-handed or right-handed ( $\mathrm{L}$ or $\mathrm{R}$ waves), according to whether the elliptical polarization of the electric vector is left-handed or right-handed about the direction of the imposed magnetic field. We shall draw diagrams for the $\mathrm{L}$ and $\mathrm{R}$ waves, respectively, but particularly for the $\mathrm{R}$ waves, we shall indicate which parts of the diagram refer to the $O$ wave and which to the $X$ wave.

\section{Dispersion Formulas}

We shall consider wave propagation in a cold magnetoplasma consisting of electrons of mass $m_{e}$ and charge $q_{e}$ vibrating with velocity $\mathbf{v}_{e}$, and ions of mass $m_{i}$ and charge $q_{i}$ vibrating with velocity $\mathbf{v}_{i}$. We shall neglect nonlinear effects and electronic collisions. Initially we shall neglect ionic collisions, but in section 7 allowance will be made for ionic collisions with neutral particles in circumstances when the neutral gas is too massive to take significant part in the wave motion. Let $\mathbf{B}_{0}$ be the imposed magnetic flux density and $\mathbf{E}$ the electric field of the wave. In the absence of collisions the equations of motion for the electrons and ions are

$$
\begin{aligned}
& m_{e} \frac{\partial \mathbf{v}_{e}}{\partial t}=q_{e}\left(\mathbf{E}+\mathbf{v}_{e} \times \mathbf{B}_{\mathbf{0}}\right) \\
& m_{i} \frac{\partial \mathbf{v}_{i}}{\partial t}=q_{i}\left(\mathbf{E}+\mathbf{v}_{i} \times \mathbf{B}_{\mathbf{0}}\right)
\end{aligned}
$$

where $t$ denotes time. The radio approximation is based on the assumption that $m_{i}$ is infinite, so that ionic motion is unimportant and (2) disappears; on the other hand, no approximation is made in (1). The hydromagnetic approximation used in this paper is based on the assumption that $m_{e}$ vanishes, so that (1) reduces to

$$
0=\mathbf{E}+\mathbf{v}_{e} \times \mathbf{B}_{0} .
$$

On the other hand no approximation is made in (2). Equation (3) implies that an electron moves in such a way as to keep the total force on it zero. For a finite electronic velocity parallel to the imposed field there can be no component of $\mathbf{E}$ in this direction. The electric field of the wave therefore vibrates in planes perpendicular to the imposed magnetic field.

\footnotetext{
${ }^{3}$ Note that Astrom [1950] reverses the names "ordinary" and "extraordinary" at hydromagnetic frequencies.
} 
To describe the propagation of a wave in the plasma the equations of motion of the electrons and ions must be combined with Maxwell's equations and with the equations of continuity. Except for propagation strictly parallel to the imposed magnetic field, waves even in a cold magnetoplasma involve compression and rarifaction of both the electrons and the ions. ${ }^{4}$ If $N_{e}$ and $N_{i}$ are the mean electron density and ion density respectively, and $\delta N_{e}$ and $\delta N_{i}$ are the corresponding fluctuations caused by the wave, the equations of continuity are

$$
\begin{aligned}
& \frac{\partial \delta N_{e}}{\partial t}=-N_{e} \nabla \cdot \mathbf{v}_{e} \\
& \frac{\partial \delta N_{i}}{\partial t}=-N_{i} \nabla \cdot \mathbf{v}_{i} .
\end{aligned}
$$

The current density $\mathbf{J}$ and the charge density $\rho$ in the plasma are given by

$$
\mathbf{J}=N_{e} q_{e} \mathbf{v}_{e}+N_{i} q_{i} \mathbf{v}_{i}
$$

and

$$
\rho=q_{e} \delta N_{e}+q_{i} \delta N_{i}
$$

It is assumed that the plasma is, on the average, neutral so that

$$
0=q_{e} N_{e}+q_{i} N_{i}
$$

The equation of continuity of charge need not be written down since it is derivable from (4) and (5) by use of (6) and (7).

To obtain the dispersion equation for a wave of angular frequency $\omega$ and propagation vector $\mathbf{k}$ we substitute into the above mentioned equations the wave function

$$
\exp \{j(\omega t-\mathbf{k} \cdot \mathbf{r})\}
$$

where $\mathbf{r}$ denotes position vector. The equations of motion (3) and (2) then become

$$
\begin{gathered}
0=\mathbf{E}+\mathbf{v}_{e} \times \mathbf{B}_{o} \\
j \omega m_{i} \mathbf{v}_{i}=q_{i}\left(\mathbf{E}+\mathbf{v}_{i} \times \mathbf{B}_{0}\right)
\end{gathered}
$$

while the equations of continuity become

$$
\begin{aligned}
& \frac{\delta N_{e}}{N_{e}}=\frac{1}{\omega} \mathbf{k} \cdot \mathbf{v}_{e} \\
& \frac{\delta N_{i}}{N_{i}}=\frac{1}{\omega} \mathbf{k} \cdot \mathbf{v}_{i} .
\end{aligned}
$$

On eliminating the magnetic field of the wave from Maxwell's equations we obtain

$$
(\mathbf{k} \cdot \mathbf{E}) \mathbf{k}-\left(k^{2}-\frac{\omega^{2}}{c^{2}}\right) \mathbf{E}=j \omega \mu_{0}\left(N_{e} q_{e} \mathbf{v}_{e}+N_{i} q_{i} \mathbf{v}_{i}\right)
$$

4 Thus equation (101) of Lighthill's treatment [1960] is inapplicable to a cold magnetoplasma and this upsets the corresponding section of his paper except for propagation strictly parallel to the imposed magnetic field. 
When required, the magnetic field $\mathbf{H}$ of the wave is given by

$$
\mathbf{H}=\frac{1}{\mu_{0} \omega} \mathbf{k} \times \mathbf{E}
$$

where $\mu_{0}$ is the vacuum inductivity. Equation (15) shows not only that the electric and magnetic fields of the wave are mutually perpendicular but also that the magnetic field of the wave vibrates in a plane perpendicular to the direction of phase propagation.

In the magnetosphere we are interested in discussing the propagation of waves above and below the following frequencies: the electronic plasma frequency $f_{N e}$, the electronic gyrofrequency $f_{M e}$, the ionic plasma frequency $f_{N i}$, and the ionic gyrofrequency $f_{M i}$. The corresponding angular frequencies will be denoted by $\omega_{N e}, \omega_{M e}, \omega_{N i}$ and $\omega_{M i}$. At hydromagnetic frequencies it is convenient to express velocities in terms of the ionic Alfvén velocity $A_{i}$ given by

$$
A_{i}=c \frac{\omega_{M i}}{\omega_{N i}}
$$

where $c$ is the velocity in free space. At whistler frequencies it is convenient to compare velocities with the electronic Alfvén velocity $A_{e}$ given by

$$
A_{e}=c \frac{\omega_{M e}}{\omega_{N e}} .
$$

Let the imposed magnetic field point in the direction of the $z$-axis, and let the direction of phase propagation lie in the $y z$ plane as shown in figure 1 . With this choice of coordinate axes we may write

$$
\begin{aligned}
\mathbf{B}_{0} & =\left(0,0, \mathbf{B}_{0}\right) \\
\mathbf{k} & =\left(0, k_{\perp}, k_{\|}\right)=k\left(0, \sin \theta_{p}, \cos \theta_{p}\right) .
\end{aligned}
$$

The angle $\theta_{p}$ is the angle between the direetion of phase propagation and the direction of the imposed magnetic field. It must be distinguished from the angle $\theta_{g}$ introduced later for the angle between the direction of group propagation and the direction of the imposed magnetic field. We

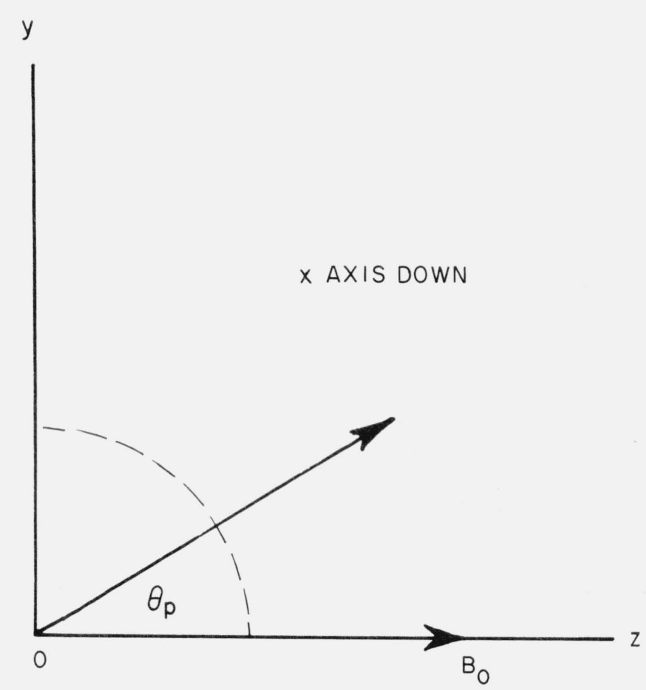

FIGURE 1. Illustrating the direction of the imposed magnetic flux density $\mathbf{B}_{0}$ and the direction of the phase propagation vector $\mathbf{k}$ in relation to the axes of coordinates. 
shall encounter situations in which the direction of phase propagation is perpendicular to the imposed magnetic field while the direction of group propagation is parallel to the imposed magnetic field.

If we substitute from (18) and (19) into (10) through (14), we obtain a set of linear algebraic equations for the ratios of the following quantities:

$$
\delta N_{e}, \delta N_{i}, E_{x}, E_{y}, v_{e x}, v_{e y}, v_{e z}, v_{i x}, v_{i y}
$$

The component of the ionic velocity in the direction of the imposed magnetic field does not appear because there is no component of the electric field in this direction to drive the ions; thus the ionic motion, which is substantially the fluid motion, is in planes perpendicular to the imposed magnetic field regardless of the direction of propagation. Elimination of the quantities (20) from the linear algebraic equations gives the dispersion relation

$$
\begin{aligned}
\cos ^{2} \theta_{p}\left(1-\frac{\omega^{2}}{\omega_{M i}^{2}}\right)\left(\frac{k c}{\omega}\right)^{4}-\left(1+\cos ^{2} \theta_{p}\right)\left(1+\frac{\omega_{M i}^{2}}{\omega_{N i}^{2}}-\frac{\omega^{2}}{\omega_{N i}^{2}}\right) \frac{\omega_{N i}^{2}}{\omega_{M i}^{2}}\left(\frac{k c}{\omega}\right)^{2} & +\left\{\left(1+\frac{\omega_{M i}^{2}}{\omega_{N i}^{2}}\right)^{2}-\frac{\omega^{2} \omega_{M i}^{2}}{\omega_{N i}^{4}}\right\} \frac{\omega_{N i}^{4}}{\omega_{M i}^{4}}=0
\end{aligned}
$$

of which the solution is

$$
\begin{aligned}
\left(\frac{k c}{\omega}\right)^{2}=1+\frac{\omega_{N i}^{2}}{\omega_{M i}^{2}} \frac{1}{1-\frac{\omega^{2}}{\omega_{M i}^{2}}}\left[1+\frac{1}{\cos ^{2} \theta_{p}}\left\{\frac{1}{2} \sin ^{2} \theta_{p}\left(1+\frac{\omega_{M i}^{2}}{\omega_{N i}^{2}}-\frac{\omega^{2}}{\omega_{N i}^{2}}\right)\right.\right. & \\
& \pm \sqrt{\left.\left.\frac{1}{4} \sin ^{4} \theta_{p}\left(1+\frac{\omega_{M i}^{2}}{\omega_{N i}^{2}}-\frac{\omega^{2}}{\omega_{N i}^{2}}\right)^{2}+\cos ^{2} \theta_{p} \frac{\omega^{2}}{\omega_{M i}^{2}}\right\}\right]} .
\end{aligned}
$$

This is the formula that, at hydromagnetic frequencies, replaces the Appleton-Hartree formula

$$
\left(\frac{k c}{\omega}\right)^{2}=\frac{\frac{\omega_{N e}^{2}}{\omega^{2}}}{1-\frac{\frac{1}{2} \frac{\omega_{M e}^{2}}{\omega^{2}} \sin ^{2} \theta_{p}}{1-\frac{\omega_{N e}^{2}}{\omega^{2}}} \pm \sqrt{\frac{1 \frac{\omega_{M e}^{4}}{4} \omega^{4}}{\left(1-\frac{\omega_{N e}^{2}}{\omega^{2}}\right)^{2}}+\cos ^{2} \theta_{p} \frac{\omega_{M e}^{2}}{\omega^{2}}}}
$$

applicable at radio frequencies. The alternative signs in (22) and (23) refer to the two magnetoionic waves.

The quantity $k c / \omega$ appearing in (21), (22), and (23) is the refractive index referred to the free space velocity $c$ in the manner customary at radio and optical frequencies. However, at hydromagnetic frequencies, it is often more convenient to use the refractive index $k A_{i} / \omega$ referred to the ionic Alfvén velocity. Furthermore, throughout the magnetosphere and in many other situations encountered in plasma physics, the ionic gyrofrequency is small compared with the ionic plasma frequency. Consequently the expression

$$
1+\frac{\omega_{M i}^{2}}{\omega_{N i}^{2}}
$$

appearing in (21) and (22) may usually be replaced by unity. Hence (21) and (22) may usually be 
replaced by

$$
\cos ^{2} \theta_{p}\left(1-\frac{\omega^{2}}{\omega_{M i}^{2}}\right)\left(\frac{k A_{i}}{\omega}\right)^{4}-\left(1+\cos ^{2} \theta_{p}\right)\left(1-\frac{\omega^{2}}{\omega_{N i}^{2}}\right)\left(\frac{k A_{i}}{\omega}\right)^{2}+\left(1-\frac{\omega^{2} \omega_{M i}^{2}}{\omega_{N i}^{4}}\right)=0\left(\omega_{M i} \ll \omega_{N i}\right)
$$

and

$$
\begin{aligned}
&\left(\frac{k A_{i}}{\omega}\right)^{2}= \frac{1}{1-\frac{\omega^{2}}{\omega_{M i}^{2}}}\left[1+\frac{1}{\cos ^{2} \theta_{p}}\left\{\frac{1}{2} \sin ^{2} \theta_{p}\left(1-\frac{\omega^{2}}{\omega_{N i}^{2}}\right)\right.\right. \\
&\left.\left. \pm \sqrt{\frac{1}{4} \sin ^{4} \theta_{p}\left(1-\frac{\omega^{2}}{\omega_{N i}^{2}}\right)^{2}+\cos ^{2} \theta_{p} \frac{\omega^{2}}{\omega_{M i}^{2}}}\right\}\right]\left(\omega_{M i} \ll \omega_{N i}\right) .
\end{aligned}
$$

Moreover, at frequencies of the order of the ionic gyrofrequency and below, (26) may be further simplified to

$$
\begin{aligned}
&\left(\frac{k A_{i}}{\omega}\right)^{2}= \frac{1}{1-\frac{\omega^{2}}{\omega_{M i}^{2}}}\left[1+\frac{1}{\cos ^{2} \theta_{p}}\left\{\frac{1}{2} \sin ^{2} \theta_{p}\right.\right. \\
&\left.\left. \pm \sqrt{\frac{1}{4} \sin ^{4} \theta_{p}+\cos ^{2} \theta_{p} \frac{\omega^{2}}{\omega_{M i}^{2}}}\right\}\right]\left(\omega \text { and } \omega_{M i} \ll \omega_{N i}\right) .
\end{aligned}
$$

When the frequency becomes small compared with the ionic gyrofrequency, the dispersion formula (27) tends to the one encountered in fluid mechanics literature on magnetohydrodynamic waves.

Virtually all the techniques that have been developed for handling the radio approximation (23) are equally applicable for studying the hydromagnetic approximation (22). In particular, the concept of approximations for quasi-longitudinal and quasi-transverse propagation that have proved convenient in handling (23) is equally applicable to (22). We have quasi-longitudinal propagation when the $\cos ^{2} \theta_{p}$ term under the radical dominates the $\sin ^{4} \theta_{p}$ term; we have quasitransverse propagation when the $\sin ^{4} \theta_{p}$ term dominates the $\cos ^{2} \theta_{p}$ term. By equating these two terms in (22) we see that, for the hydromagnetic approximation, the transition from quasilongitudinal to quasi-transverse propagation occurs where

$$
\frac{\sin ^{2} \theta_{p}}{2 \cos \theta_{p}}=\frac{\frac{\omega}{\omega_{M i}}}{\left|1+\frac{\omega_{M i}^{2}}{\omega_{N i}^{2}}-\frac{\omega^{2}}{\omega_{N i}^{2}}\right|} .
$$

If expression (24) can be replaced by unity, (28) becomes

$$
\frac{\sin ^{2} \theta_{p}}{2 \cos \theta_{p}}=\frac{\frac{\omega}{\omega_{M i}}}{\left|1-\frac{\omega^{2}}{\omega_{N i}^{2}}\right|} \quad\left(\omega_{M i} \ll \omega_{N i}\right) .
$$

Other useful approximations to (28) are

$$
\frac{\sin ^{2} \theta_{p}}{2 \cos \theta_{p}}=\frac{\omega_{N i}^{2}}{\omega \omega_{M i}} \quad\left(\omega \gg \omega_{N i} \text { and } \omega_{M i}\right)
$$

and

$$
\frac{\sin ^{2} \theta_{p}}{2 \cos \theta_{p}}=\frac{\omega}{\omega_{M i}} \quad\left(\omega \text { and } \omega_{M i} \ll \omega_{N i}\right) .
$$


FIGURE 2. Illustrating the distribution between quasilongitudinal and quasi-transverse propagation in the magnetosphere roughly one earth's radius out in the equatorial plane.

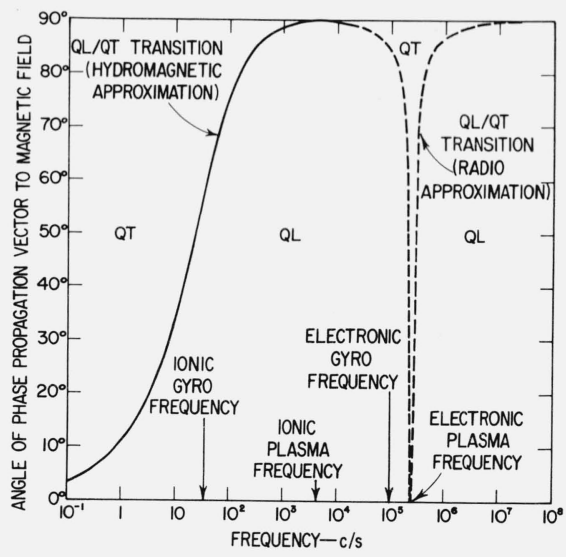

For the radio approximation (23) the transition from quasi-longitudinal to quasi-transverse propagation occurs where

$$
\frac{\sin ^{2} \theta_{p}}{2 \cos \theta_{p}}=\frac{\left|1-\frac{\omega_{N e}^{2}}{\omega^{2}}\right|}{\frac{\omega_{M e}}{\omega}}
$$

to which the low frequency approximation is

$$
\frac{\sin ^{2} \theta_{p}}{2 \cos \theta_{p}}=\frac{\omega_{N e}^{2}}{\omega \omega_{M e}} \quad\left(\omega \ll \omega_{N e}\right) .
$$

It should be noted that the expressions quasi-longitudinal and quasi-transverse refer to the direction of propagation and not to the direction of vibration of particles. It should further be noted that these expressions refer to the direction of phase propagation in relation to the imposed magnetic field and not to the direction of group propagation. Situations will occur in which propagation is quasi-transverse even though the direction of group propagation is nearly parallel to the imposed magnetic field.

To obtain some idea when propagation is quasi-longitudinal and when quasi-transverse, let us apply (28) through (33) to a magnetoplasma of the type existing in the magnetosphere roughly one earth's radius out in the equatorial plane. The frequencies $f_{M i}, f_{N i}, f_{M e}$, and $f_{N e}$ then have values such as those shown in figure 2. The continuous curve in figure 2 is drawn from (28) and the broken curve from (32). We notice how well the hydromagnetic approximation joins onto the radio approximation. The reason is that (30) and (33) are numerically identical because the ratio of the square of the plasma frequency to the gyrofrequency is independent of particle mass. Figure 2 illustrates the well-known fact that, in the radio range, propagation is mainly quasi-longitudinal except near the electronic plasma frequency. Figure 2 also shows that, in the hydromagnetic range, propagation is principally quasi-transverse below the ionic gyrofrequency and principally quasi-longitudinal above. The usual dispersion equation for magnetohydrodynamic waves given in the fluid mechanics literature is in fact a quasi-transverse approximation, and this is why such papers sometimes make no reference to circularly polarized waves.

We have seen in figure 2 that the hydromagnetic approximation fits on to the radio approximation remarkably well, and we need to investigate to what extent this convenient result is generally applicable. For frequencies high compared with the ionic gyro and plasma frequencies (22) 
may be written

$$
\left(\frac{k c}{\omega}\right)^{2}=1-\frac{\frac{\omega_{N i}^{4}}{\omega^{2} \omega_{M i}^{2}}}{\frac{1}{2} \sin ^{2} \theta_{p} \mp \sqrt{\frac{1}{4} \sin ^{4} \theta_{p}+\cos ^{2} \theta_{p} \frac{\omega_{N i}^{4}}{\omega^{2} \omega_{M i}^{2}}}}
$$

while, for frequencies small compared with the electronic plasma frequency, (23) may be written

$$
\left(\frac{k c}{\omega}\right)^{2}=1-\frac{\frac{\omega_{N e}^{4}}{\omega^{2} \omega_{M e}^{2}}}{\frac{\omega_{N e}^{2}}{\omega_{M e}^{2}}+\frac{1}{2} \sin ^{2} \theta_{p} \mp \sqrt{\frac{1}{4} \sin ^{4} \theta_{p}+\cos ^{2} \theta_{p} \frac{\omega^{4}{ }_{N e}^{2} \omega_{M e}^{2}}{\omega^{2}}}} \quad\left(\omega \ll \omega_{N e}\right) .
$$

Since

$$
\frac{\omega_{N i}^{2}}{\omega_{M i}}=\frac{\omega_{N e}^{2}}{\omega_{M e}}
$$

(34) and (35) would be completely identical were it not for the term $\omega_{N e}^{2} / \omega_{M e}^{2}$ in the denominator in (35). Unfortunately, in the magnetosphere, the electronic plasma frequency is not small compared with the electronic gyrofrequency, and so the additional term in (35) as compared with (34) is significant. However, this is only true for quasi-transverse propagation, which, as indicated in figure 2 , is comparatively unimportant at the frequency where the transition is made from the hydromagnetic approximation to the radio approximation. For quasi-longitudinal conditions at the transition frequency the $\cos ^{2} \theta_{p}$ term under the radical in (35) dominates the $\sin ^{4} \theta_{p}$ term, so that (34) and (35) are identical if the transition frequency is small compared with the electronic gyrofrequency. In fact, for quasi-longitudinal propagation, (34) and (35) reduce to

$$
\begin{array}{ll}
\left(\frac{k c}{\omega}\right)^{2}=1 \pm \frac{\omega_{N i}^{2}}{\omega \omega_{M i} \cos \theta_{p}} & (\mathrm{QL}) \\
\left(\frac{k c}{\omega}\right)^{2}=1 \pm \frac{\omega_{N e}^{2}}{\omega \omega_{M e} \cos \theta_{p}} & (\mathrm{QL})
\end{array}
$$

and the identity of these equations follows from (36). It is upon (38) that the Eckersley's law for whistlers is based. Equations (37) and (38) show that, while the basis of the Eckersley law is usually thought of as the low frequency behavior of the radio approximation, it may equally well be thought of as the high frequency behavior of the hydromagnetic approximation. Thus in all circumstances where there is an interval of frequency in which the Eckersley law for whistlers is applicable, the hydromagnetic approximation is bound to fit smoothly onto the radio approximation.

The fit between the hydromagnetic and radio approximations is illustrated in figure 3 for longitudinal propagation of the $\mathrm{R}$ wave and for the same ionization density and imposed magnetic field used in figure 2. The continuous curve in figure 3 shows the dispersion behavior for the $R$ wave using the full cold plasma theory. The broken curve in figure 3 shows the Eckersley law, and we see how this agrees with the full theory at audio frequencies. If the dispersion curve were plotted entirely on the basis of the radio approximation it would follow the continuous curve in figure 3 from whistler frequencies upwards but the broken curve from whistler frequencies downwards. On the other hand if the dispersion curve were plotted entirely from the hydromagnetic approximation it would follow the continuous curve in figure 3 from whistler frequencies down- 


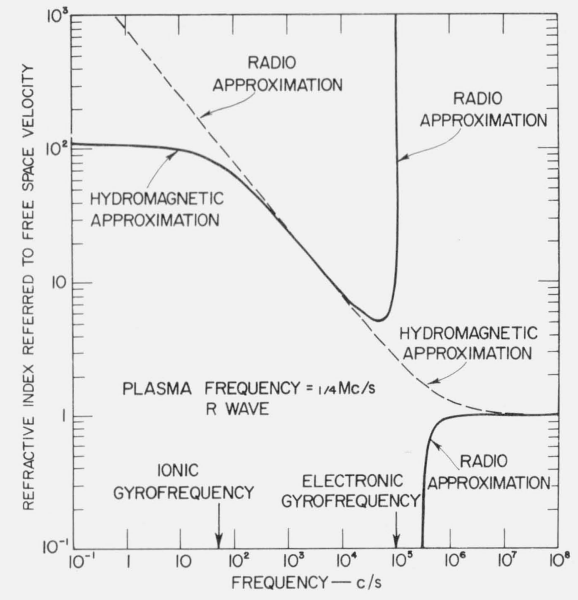

Figure 3. Illustrating the fit between the hydromagnetic and radio approximation for longitudinal propagation of the $R$ wave about one earth's radius out in the equatorial plane.
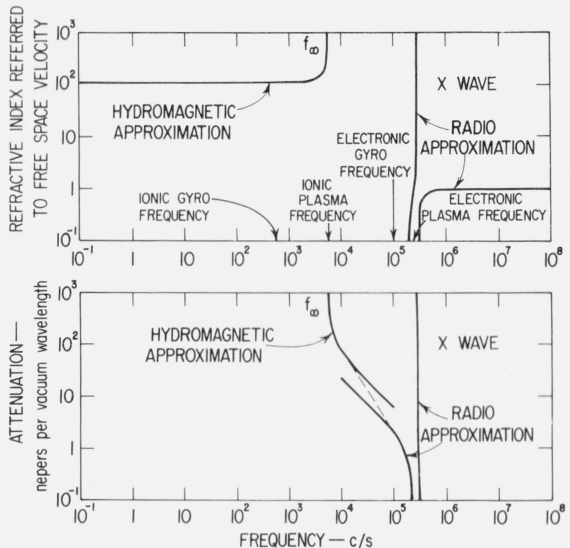

FiguRE 4. Illustrating propagation of the $X$ wave transverse to the earth's magnetic field about one earth's radius out in the equatorial plane.

wards but the broken curve from whistler frequencies upwards. Thus figure 3 illustrates for longitudinal propagation how the hydromagnetic approximation fits onto the radio approximation at whistler frequencies where both approximations give the Eckersley law.

On the other hand, when the direction of phase propagation is transverse to the imposed magnetic field, there is misfit between the hydromagnetic and radio approximations at whistler frequencies on account of the additional term in the denominator of (35) not present in the denominator of (34). This misfit is illustrated in figure 4 for the ionization density and imposed magnetic field used in figure 2. Figure 4 shows that, for propagation of the $\mathrm{X}$ wave transverse to the imposed magnetic field, there is a stop-band extending from the lower critical frequency in the radio range down to a frequency of $f_{\infty}$ close to the ionic plasma frequency. The misfit occurs in this stop-band and is indicated by the broken curve joining the two approximations. It should be mentioned that the lower edge of the stop-band in figure 4 does not occur exactly at the ionic plasma frequency. Furthermore, the effect of the misfit between the hydromagnetic and radio approximations shifts the low frequency limit of the stop-band downwards to some extent; indeed, far out in the magnetosphere, the shift is as much as a decade. It is feasible to modify the hydromagnetic approximation to avoid this misfit, but this modification is not discussed in this paper.

The hydromagnetic and radio approximations cover almost everything required for propagation in the magnetosphere regarded as a cold plasma, except for quasi-transverse propagation at whistler frequencies. This exception is quite restricted as shown in figure 2. Virtually everything of interest in the cold plasma theory of wave propagation in the magnetosphere is therefore obtained by combining the Appleton-Hartree formula at radio frequencies with the hydromagnetic approximation given by (22).

\section{Dispersion Curves}

To illustrate the frequency behavior of the hydromagnetic dispersion formula (22), it will be necessary to assume a numerical value for the ratio of ionic plasma frequency to ionic gyrofrequency. In the magnetosphere this ratio varies from about 50 to 1,000. Use of too large a value of this ratio is inconvenient, however, as some of the phenomena to be illustrated occur for values 
of $\theta_{p}$ between $\tan ^{-1} \omega_{N i} / \omega_{M i}$ and $\frac{1}{2} \pi$. We shall therefore use for purposes of illustration the value

$$
\frac{\omega_{N i}}{\omega_{M i}}=\frac{c}{A_{i}}=10.7
$$

which corresponds to

$$
\frac{\omega_{N e}}{\omega_{M e}}=\frac{c}{A_{e}}=\left\{\begin{array}{l}
1 / 4 \text { (hydrogen) } \\
1 / 8 \text { (helium) } \\
1 / 16 \text { (atomic oxygen) }
\end{array}\right.
$$

However, the curves will be plotted so as to be independent of the value of the ionic plasma frequency for wave frequencies of the order of the ionic gyrofrequency and below. This is achieved, in accordance with (27), by referring the refractive index not to the free space velocity but to the ionic Alfvén velocity.

For the numerical values given in (39) and (40), the transition between quasi-longitudinal and quasi-transverse propagation is illustrated in figure 5 on the basis of (28). In figure $5 \mathrm{a}$ eq. (28) may in fact be replaced by (31), and in figure $5 b$ by (29). Figure 5 b illustrates the wide range of conditions for which propagation is quasi-longitudinal. Moreover, as the ratio of the ionic plasma frequency to the ionic gyrofrequency increases above the value given in (39), the regions of quasitransverse propagation above the ionic gyrofrequency become even more restricted than is shown in figure 5b. On the other hand, for frequencies of the order of the ionic gyrofrequency and below, the behavior is independent of the value of the ionic plasma frequency as shown by (31) and figure 5a. As the frequency tends to zero, propagation becomes predominantly quasi-transverse no matter how large the value of $\omega_{N i} / \omega_{M i}$.
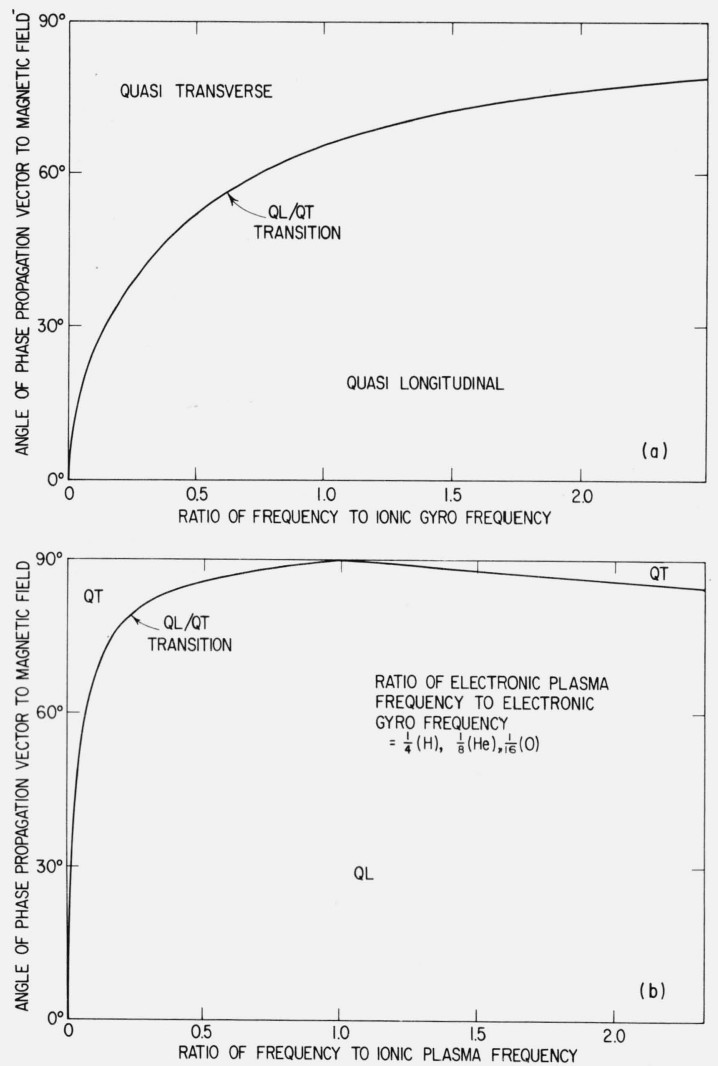

FiguRE 5. Illustrating for the hydromagnetic approximation the transition from quasi-longitudinal to quasitransverse propagation (a) on the scale of the ionic gyrofrequency and $(b)$ on the scale of the ionic plasma frequency. 
To study the variation of refractive index with frequency it is convenient initally to examine the situations in which the refractive index for one of the waves is either zero or infinite, or in which the refractive indices for the two waves are identical. The zeros of refractive index are obtained by equating to zero the term in (21) not involving $k c / \omega$. The frequency derived is outside the range of validity of the hydromagnetic approximation under conditions of interest in the magnetosphere. Zeros of refractive index occur in the range of validity of the radio approximation and are given by the well-known ionospheric formulas for critical frequency. For the magnetosphere there are no zeros of refractive index at hydromagnetic frequencies.

Besides the infinity of refractive index occurring at radio frequencies, there are infinities occurring at hydromagnetic frequencies. Infinities of refractive index for the hydromagnetic approximation are obtained by equating to zero the coefficient of $(k c / \omega)^{4}$ in $(21)$. We see that there is an infinity of refractive index when $\omega=\omega_{M i}$ and when $\theta_{p}=1 / 2 \pi$. Thus, for the hydromagnetic approximation, the refractive index of one of the waves is infinite for all frequencies when the direction of phase propagation is transverse to the imposed magnetic field. This is in fact the $\mathrm{O}$ wave for which transverse propagation is independent of the imposed magnetic field. For transverse propagation the $\mathrm{O}$ wave has a stop-band extending from the electronic plasma frequency indefinitely downwards. At hydromagnetic frequencies this wave is highly evanescent and has a refractive index on the imaginary axis close to the point at infinity. This becomes identical with the point at infinity for the hydromagnetic approximation because of the neglect of electron inertia. Thus, by putting $\theta_{p}=1 / 2 \pi$ in (21), we see that the refractive indices of the $\mathrm{O}$ and $\mathrm{X}$ waves for strictly transverse propagation using the hydromagnetic approximation are

$$
\left(\frac{k c}{\omega}\right)^{2}=\infty \quad(\mathrm{T}, \mathrm{O})
$$

and

$$
\left(\frac{k c}{\omega}\right)^{2}=\frac{\omega_{N i}^{2}}{\omega_{M i}^{2}} \frac{\left(1+\frac{\omega_{M i}^{2}}{\omega_{N i}^{2}}\right)^{2}-\frac{\omega^{2} \omega_{M i}^{2}}{\omega_{N i}^{4}}}{1+\frac{\omega_{M i}^{2}}{\omega_{N i}^{2}}-\frac{\omega^{2}}{\omega_{N i}^{2}}} \quad(\mathrm{~T}, \mathrm{X})
$$

It is from (42) that the hydromagnetic approximation shown in figure 4 is plotted. For transverse propagation of the $\mathrm{X}$ wave there is an infinity of refractive index for the hydromagnetic approximation at the frequency $f_{\infty}$ (angular frequency $\omega_{\infty}$ ) for which the denominator in (42) vanishes. Hence

$$
\omega_{\infty}=\left(\omega_{N i}^{2}+\omega_{M i}^{2}\right)^{1 / 2}
$$

which reduces to $\omega_{\infty}=\omega_{N i}$ when $\omega_{M i} \ll \omega_{N i}$. As mentioned in connection with figure 4 misfit between the hydromagnetic and radio approximations for quasi-transverse propagation at whistler frequencies depresses the value of $\omega_{\infty}$ below that given by (43). When the effect of electron inertia at the ionic plasma frequency is taken into account Stix [1962] gives for $\omega_{\infty}$ the approximate equation

$$
\frac{1}{\omega_{\infty}^{2}}=\frac{1}{\omega_{N i}^{2}+\omega_{M i}^{2}}+\frac{1}{\omega_{M i} \omega_{M e}}
$$

which may usually be written in the form

$$
\frac{1}{\omega_{\infty}^{2}}=\frac{1}{\omega_{N i}^{2}}+\frac{1}{\omega_{M i} \omega_{M e}}\left(\omega_{M i} \ll \omega_{N i}\right) .
$$

For the curves drawn in this paper the frequency $\omega_{\infty}$ will be indistinguishable from $\omega_{N i}$, but it should be remembered that $\omega_{\infty}$ is given more generally by (45). The difference is numerically important 
at the higher levels in the magnetosphere and also in laboratory plasma physics. This feature has been studied by C. O. Hines [1957] and by Auer, Hurwitz, and Miller [1958].

Before actually drawing dispersion curves it is important to notice the conditions under which the refractive indices for the two waves are identical. For the hydromagnetic approximation this happens when the quantity under the radical in (22) vanishes, that is, when $\omega=\omega_{\infty}$ and $\theta_{p}=1 / 2 \pi$. This is simply the situation in which the expression on the right-hand side of (42) is infinite and is consequently identical with that on the right-hand side of (41). As is well known for the radio approximation, situations where the refractive indices of two waves are identical lead to coupling between the waves and to interchange in the names of the waves. This behavior for the hydromagnetic approximation is conveniently illustrated by plotting the inverse of the square of the refractive index versus frequency as shown in figure 6 . The continuous and broken curves for $\theta_{p}=90^{\circ}$ in figure 6 are based on (41) and (42), respectively and we notice that they intersect near the ionic plasma frequency. As we depart from strictly transverse propagation, the curves for the $\mathrm{O}$ wave become connected to the curves for the $\mathrm{X}$ wave in the manner illustrated. The sharp change as we move away from strictly transverse propagation conditions becomes a continuous but rapid change if electron inertia is taken into account or if ionic collisions are taken into account. The behavior is qualitatively similar to that encountered in the radio approximation close to strictly longitudinal propagation conditions at the electronic plasma frequency [Booker, 1934].

We are now in a position to replot figure 6 to give the dependence of refractive index upon frequency. This is done in figure 7. From figure $7 \mathrm{~b}$ we see that the $\mathrm{R}$ wave is the $\mathrm{X}$ wave for $f<f_{\infty}$ and the $\mathrm{O}$ wave for $f>f_{\infty}$. From figure 7 a we see that the $\mathrm{L}$ wave has a hydromagnetic pass-band extending from zero frequency to the ionic gyrofrequency and that, in this band, the $\mathrm{L}$ wave is also the $\mathrm{O}$ wave. Near the low frequency end of this band the $\mathrm{L}$ wave is the wave originally introduced by Alfvén [1950], while near the high frequency end of the pass-band the $\mathrm{L}$ wave is what is known to laboratory plasma physicists as the ion cyclotron wave [Stix, 1962].

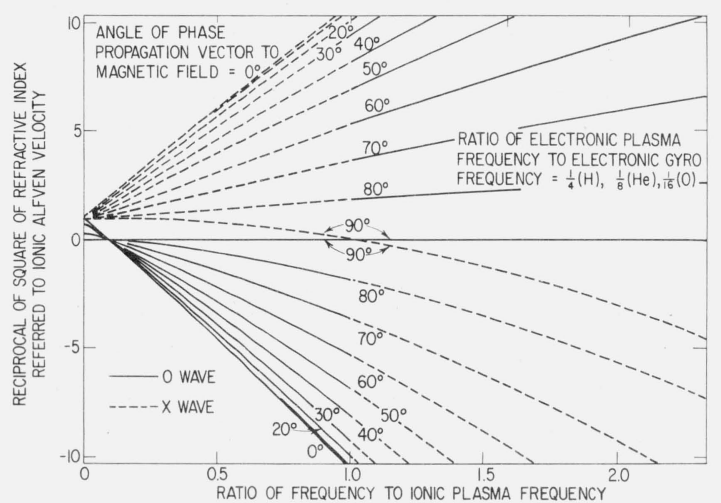

FIGURE 6. Illustrating the exchange in the nomenclature of the $O$ and $X$ waves near the ionic plasma frequency.
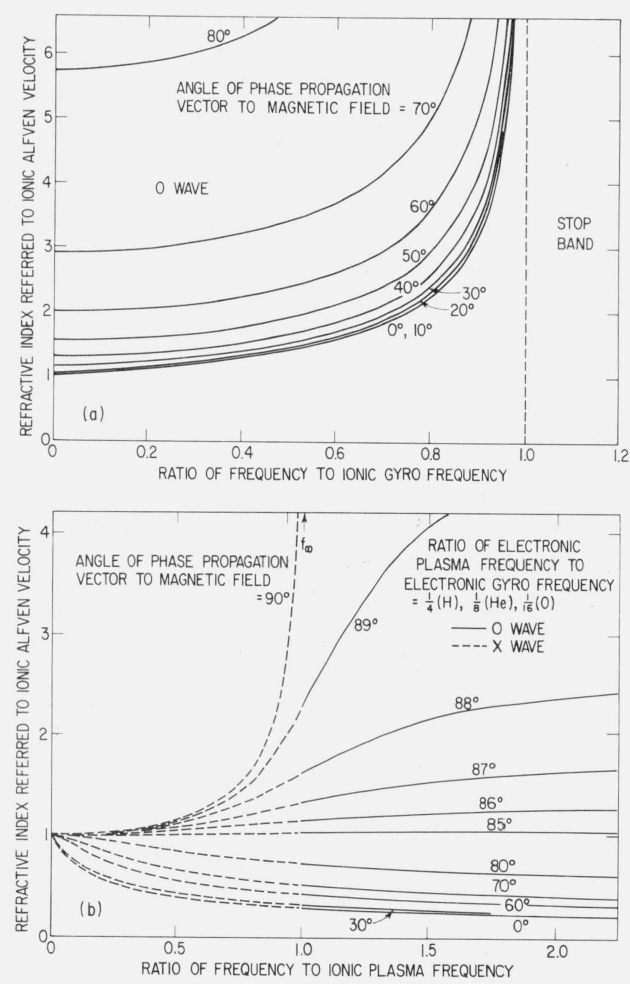

FiguRE 7. Illustrating, for the hydromagnetic approximation, refractive index versus frequency (a) for the $L$ wave and (b) for the $R$ wave. 
Various sections of the dispersion curves shown in figure 7 (and of subsequent curves) may be conveniently approximated by using the quasi-longitudinal and quasi-transverse approximations. When the $\cos ^{2} \theta_{p}$ term under the radical dominates the $\sin ^{4} \theta_{p}$ term, (22) reduces to the quasilongitudinal approximation

$$
\left(\frac{k c}{\omega}\right)^{2}=1+\frac{\omega_{N i}^{2}}{\omega_{M i}^{2}} \frac{1 \pm \frac{\omega}{\omega_{M i} \cos \theta_{p}}}{1-\frac{\omega^{2}}{\omega_{M i}^{2}}} \quad(\mathrm{QL})
$$

where the upper sign refers to the $\mathrm{L}$ wave and lower sign to the $\mathrm{R}$ wave. $\mathrm{By}$ putting $\theta_{p}=0$, we deduce for strictly longitudinal propagation using the hydromagnetic approximation

$$
\left(\frac{k c}{\omega}\right)^{2}=1+\frac{\omega_{N i}^{2}}{\omega_{M i}^{2}} \frac{1}{1 \mp \frac{\omega}{\omega_{M i}}}
$$

When the $\sin ^{4} \theta_{p}$ term under the radical in (22) dominates the $\cos ^{2} \theta_{p}$ term, we obtain for the quasitransverse approximation for the $\mathrm{O}$ wave

$$
\left(\frac{k c}{\omega}\right)^{2}=1+\frac{\omega_{N i}^{2}}{\omega_{M i}^{2}} \frac{1+\left(1+\frac{\omega_{M i}^{2}}{\omega_{N i}^{2}}-\frac{\omega^{2}}{\omega_{N i}^{2}}\right) \tan ^{2} \theta_{p}}{1-\frac{\omega^{2}}{\omega_{M i}^{2}}}(\mathrm{QT}, \mathrm{O})
$$

of which useful further approximations are

$$
\left(\frac{k c}{\omega}\right)^{2}=\left\{\begin{array}{l}
\frac{\omega_{N i}^{2}}{\omega_{M i}^{2}} \frac{\sec ^{2} \theta_{p}}{1-\frac{\omega^{2}}{\omega_{M i}^{2}}}\left(\mathrm{QT}, \mathrm{O} ; \omega \text { and } \omega_{M i} \ll \omega_{N i}\right) \\
\sec ^{2} \theta_{p} \quad\left(\mathrm{QT}, \mathrm{O} ; \omega \gg \omega_{M i} \text { and } \omega_{N i}\right) .
\end{array}\right.
$$

To obtain the quasi-transverse approximation for the $\mathrm{X}$ wave the radical in (22) must be expanded by the binomial theorem, and we obtain

$$
\left(\frac{k c}{\omega}\right)^{2}=1+\frac{\omega_{N i}^{2}}{\omega_{M i}^{2}} \frac{1}{1-\frac{\omega^{2}}{\omega_{M i}^{2}}}\left[1-\frac{\frac{\omega^{2}}{\omega_{M i}^{2}}}{1+\frac{\omega_{M i}^{2}}{\omega_{N i}^{2}}-\frac{\omega^{2}}{\omega_{N i}^{2}}} \frac{1}{\sin ^{2} \theta_{p}}\right](\mathrm{QT}, \mathrm{X})
$$

which may also be written as

$$
\left(\frac{k c}{\omega}\right)^{2}=\frac{\omega_{N i}^{2}}{\omega_{M i}^{2}} \frac{\left(1+\frac{\omega_{M i}^{2}}{\omega_{N i}^{2}}\right)^{2}-\frac{\omega^{2} \omega_{M i}^{2}}{\omega_{N i}^{4}}}{1+\frac{\omega_{M i}^{2}}{\omega_{N i}^{2}}-\frac{\omega^{2}}{\omega_{N i}^{2}}}-\frac{\frac{\omega^{2}}{\omega_{M i}^{2}}}{\left(1-\frac{\omega^{2}}{\omega_{M i}^{2}}\right)\left(1+\frac{\omega_{M i}^{2}}{\omega_{N i}^{2}}-\frac{\omega^{2}}{\omega_{N i}^{2}}\right)} \frac{1}{\tan ^{2} \theta_{p}} \quad(\mathrm{QT}, \mathrm{X})
$$

From (48) and (52) we easily verify the truth of (41) and (42) when the direction of phase propagation is strictly transverse to the imposed magnetic field. 
As an example of the utility of the quasi-longitudinal and quasi-transverse approximations, consider the curve for $\theta_{p}=60^{\circ}$ in figure $7 \mathrm{~b}$. From figure $5 \mathrm{~b}$ we see that, over most of the relevant frequency range, propagation is quasi-longitudinal. Thus much of the $60^{\circ}$ curve in figure $7 \mathrm{~b}$ is represented by (46) using the lower sign. On the other hand, for the $88^{\circ}$ curve in figure $7 \mathrm{~b}$, we see from figure $5 \mathrm{~b}$ that propagation passes from quasi-transverse at sufficiently low frequencies to quasi-transverse at sufficiently high frequencies through an intermediate frequency range of quasi-longitudinal propagation. The corresponding sections of the $88^{\circ}$ curve in figure $7 \mathrm{~b}$ may therefore be sketched in from (51), (48), and (46) using the lower sign. In particular, the value to which the $88^{\circ}$ curve in figure $7 \mathrm{~b}$ is leveling off as the frequency moves above the ionic plasma frequency is given by (50).

\section{Group Propagation}

We now wish to examine group propagation for the hydromagnetic approximation and to see how it fits in with group propagation for the radio approximation. We are here considering a situation in which a pulse whose spectrum is principally confined to a limited band of frequencies is radiated into a homogeneous magnetoplasma from a source of limited dimensions near the origin. The radiated pulse may be regarded as an aggregate of wave packets radiated from the origin in different directions with different group velocities. At any time subsequent to radiation these wave packets lie along a surface that constitutes the directional pulse pattern of the source in the magnetoplasma. We wish to understand how the directional pulse patterns for both magnetoionic waves vary with frequency from radio frequencies down to frequencies small compared with the ionic gyrofrequency.

Application of the principle of stationary phase to the wave function (9) gives

$$
\frac{\mathbf{r}}{t}=\frac{\partial \omega}{\partial \mathbf{k}}
$$

which shows that the wave packets travel radially outwards from the origin with the group velocity

$$
\mathbf{V}_{g}=\frac{\partial \omega}{\partial \mathbf{k}}
$$

We shall plot polar diagrams of the group velocity vector $\mathbf{V}_{g}$ for both magnetoionic waves and for a series of frequencies. Equation (54) shows that, if the dispersion equation is regarded as defining in $\mathbf{k}$ space surfaces of constant $\omega$, then the gradient of the contour markings on these surfaces gives the group velocity in magnitude and direction. For this purpose it is convenient to write the dispersion equation (21) as

$$
\cos ^{2} \theta_{p}\left(1-\frac{\omega^{2}}{\omega_{M i}^{2}}\right)\left(\frac{k c}{\omega_{N i}}\right)^{4}-\left(1+\cos ^{2} \theta_{p}\right)\left(1+\frac{\omega_{M i}^{2}}{\omega_{N i}^{2}}-\frac{\omega^{2}}{\omega_{N i}^{2}}\right) \frac{\omega^{2}}{\omega_{M i}^{2}}\left(\frac{k c}{\omega_{N i}}\right)^{2}+\left\{\left(1+\frac{\omega_{M i}^{2}}{\omega_{N i}^{2}}\right)^{2}-\frac{\omega^{2} \omega_{M i}^{2}}{\omega_{N i}^{4}}\right\} \frac{\omega^{4}}{\omega_{M i}^{4}}=0 .
$$

If we regard $\left(k, \theta_{p}\right)$ as polar coordinates, (55) gives a curve for each value of $\omega$. These curves are shown in figure 8 for the $L$ wave and in figure $9 a$ for the $R$ wave. If we take the mirror image of these diagrams in the axes and rotate about the imposed magnetic field, we obtain surfaces with contour markings whose gradient gives the group velocity vector in accordance with (54). In figure 8 the transition between quasi-longitudinal and quasi-transverse propagation is shown, and we notice that the arrangement of straight parallel contours familiar in the fluid mechanics literature concerning magnetohydrodynamic waves is restricted to the quasi-transverse region. 


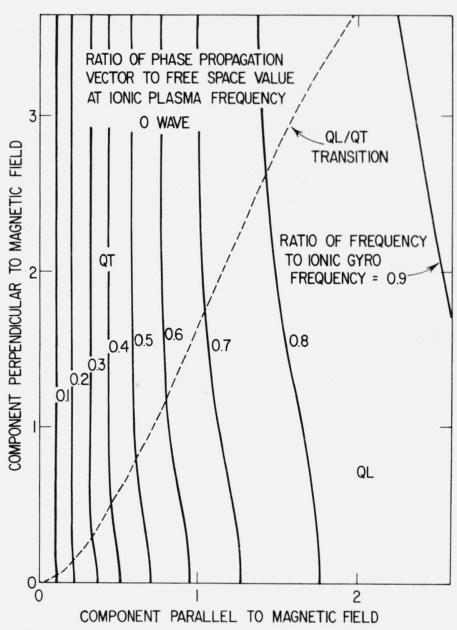

FIGURE 8. Illustrating, for the L wave, surfaces of constant $\omega$ in $\mathbf{k}$ space using the hydromagnetic approximation.
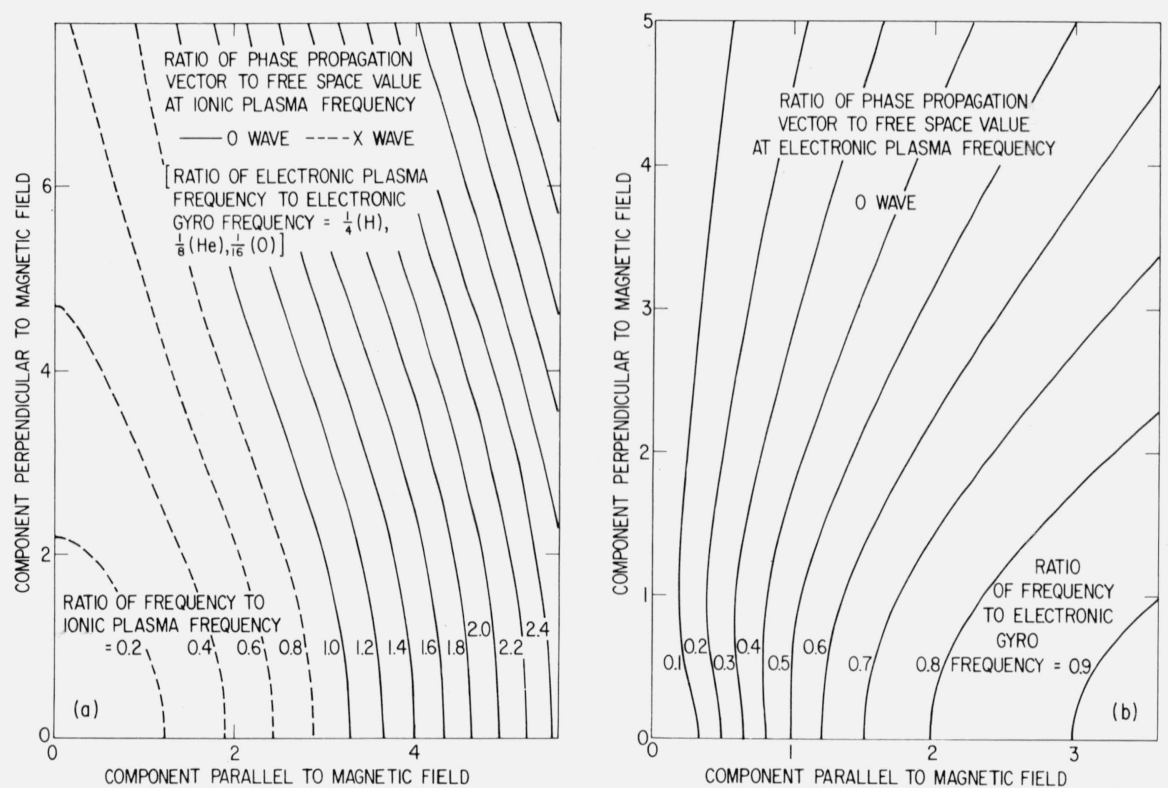

FigURE 9. Illustrating, for the $R$ wave, surfaces of constant $\omega$ in $\mathbf{k}$ space (a) using the hydromagnetic approximation on the scale of the ionic plasma frequency and (b) using the radio approximation on the scale of the electronic gyrofrequency.

In figure 9a the broken contours, unlike the continuous contours, form oval curves when the other three quadrants are drawn. In figure 9a the propagation is quasi-longitudinal over the entire diagram except for a narrow strip along the perpendicular axis. The way in which the hydromagnetic approximation for the $\mathrm{R}$ wave shown in figure $9 \mathrm{a}$ fits onto the radio approximation may be seen from figure 9b. Figure $9 \mathrm{~b}$ illustrates the whistler dispersion formula

$$
\left(\frac{k c}{\omega_{N e}}\right)^{2}=\frac{\frac{\omega}{\omega_{M e}}}{\cos \theta_{p}-\frac{\omega}{\omega_{M e}}}
$$


and is based on the work of R. L. Smith [1960] and R. Gendrin [1960]. Whereas figure 9a is drawn on the scale of the ionic plasma frequency, figure $9 \mathrm{~b}$ is drawn on the scale of the electronic gyrofrequency. To fit the two diagrams together the contour map in figure $9 \mathrm{a}$ must be appropriately contracted in scale and fitted between the perpendicular axis and the $0.1 f_{M e}$ contour in figure $9 \mathrm{~b}$.

We use the contour maps in figures 8 and 9 in the following way. If we are interested in radiation of a wave packet from the origin in a particular direction at a particular frequency, we mark the point on the appropriate contour where this direction intersects the contour at right angles. The gradient indicated by the contour map at this point then gives the group velocity in magnitude and direction in accordance with (54). The position vector from the origin to the marked point gives the direction of motion of individual wave crests within the pulse and their phase propagation constant. This procedure may be carried out analytically by writing the components of (54) parallel and perpendicular to the imposed magnetic field as

$$
\begin{aligned}
& V_{\|}=\frac{\partial \omega}{\delta k_{\|}} \\
& V_{\perp}=\frac{\partial \omega}{\delta k_{\perp}} .
\end{aligned}
$$

The derivatives are calculated by differentiating the dispersion equation (55) after converting from polar coordinates $\left(k, \theta_{p}\right)$ to Cartesian coordinates $\left(k_{\|}, k_{\perp}\right)$ in accordance with (19). If we reconvert after performing the differentiations, we obtain

$$
\begin{aligned}
\left.\frac{V_{g \|}}{A_{i}}=\frac{\frac{k c}{\omega_{N i}} \cos \theta_{p}\left[\left(1+\cos ^{2} \theta_{p}\right)\left(1-\frac{\omega^{2}}{\omega_{M i}^{2}}\right)\left(\frac{k c}{\omega_{N i}}\right)^{2}-2\left(1+\frac{\omega_{M i}^{2}}{\omega_{N i}^{2}}-\frac{\omega^{2}}{\omega_{N i}^{2}}\right) \frac{\omega^{2}}{\omega_{M i}^{2}}\right]}{\frac{\omega}{\omega_{M i}}\left[\cos ^{2} \theta_{p}\left(\frac{k c}{\omega_{N i}}\right)^{4}+\left(1+\cos ^{2} \theta_{p}\right)\left(1+\frac{\omega_{M i}^{2}}{\omega_{N i}^{2}}-2 \frac{\omega^{2}}{\omega_{N i}^{2}}\right)\left(\frac{k c}{\omega_{N i}}\right)^{2}\right.}-\left\{2\left(1+\frac{\omega_{M i}^{2}}{\omega_{N i}^{2}}\right)^{2}-3 \frac{\omega^{2} \omega_{M i}^{2}}{\omega_{N i}^{4}}\right\} \frac{\omega^{2}}{\omega_{M i}^{2}}\right] \\
\frac{V_{g_{\perp}}}{A_{i}}=\frac{\frac{k c}{\omega_{N i}} \sin \theta_{p}\left[\cos ^{2} \theta_{p}\left(1-\frac{\omega^{2}}{\omega_{M i}^{2}}\right)\left(\frac{k c}{\omega_{N i}}\right)^{2}-\left(1+\frac{\omega_{M i}^{2}}{\omega_{N i}^{2}}-\frac{\omega^{2}}{\omega_{N i}^{2}}\right) \frac{\omega^{2}}{\omega_{M i}^{2}}\right]}{\frac{\omega_{M i}}{\omega^{2}}\left[\cos ^{2} \theta_{p}\left(\frac{k c}{\omega_{N i}}\right)^{4}+\left(1+\cos ^{2} \theta_{p}\right)\left(1+\frac{\omega_{M i}^{2}}{\left.\omega_{N i}^{2}-2 \frac{\omega^{2}}{\omega_{N i}^{2}}\right)\left(\frac{k c}{\omega_{N i}}\right)^{2}}\right.\right.} \\
-\left\{2\left(1+\frac{\omega_{M i}^{2}}{\omega_{N i}^{2}}\right)^{2}-3 \frac{\omega^{2} \omega_{M i}^{2}}{\left.\left.\omega_{N i}^{4}\right\} \frac{\omega^{2}}{\omega_{M i}^{2}}\right] .}\right.
\end{aligned}
$$

To calculate the angle $\theta_{g}$ between the direction of motion of a wave packet and the direction of the imposed magnetic field we divide (60) by (59), thereby obtaining

$$
\tan \theta_{g}=\tan \theta_{p} \frac{\cos ^{2} \theta_{p}\left(1-\frac{\omega^{2}}{\omega_{M i}^{2}}\right)\left(\frac{k c}{\omega_{N i}}\right)^{2}-\left(1+\frac{\omega_{M i}^{2}}{\omega_{N i}^{2}}-\frac{\omega^{2}}{\omega_{N i}^{2}}\right) \frac{\omega^{2}}{\omega_{M i}^{2}}}{\left(1+\cos ^{2} \theta_{p}\right)\left(1-\frac{\omega^{2}}{\omega_{M i}^{2}}\right)\left(\frac{k c}{\omega_{N i}}\right)^{2}-2\left(1+\frac{\omega_{M i}^{2}}{\omega_{N i}^{2}}-\frac{\omega^{2}}{\omega_{N i}^{2}}\right) \frac{\omega^{2}}{\omega_{M i}^{2}}} .
$$

This equation relates the direction of group propagation to the direction of phase propagation and is illustrated graphically in figure 10. Using this relationship we may express the components of the group velocity vector in (59) and (60) as functions of $\theta_{g}$. If the relation between group velocity and the angle $\theta_{g}$ is illustrated in polar form, we obtain the diagrams shown in figure 11 . In figure 11 the mirror image in the perpendicular axis should also be drawn and each pattern should be rotated round the imposed magnetic field. For a pulse radiated from the origin at time zero each diagram in figure 11 gives the radiated pulse pattern at unit time. Pulse patterns at a succession 

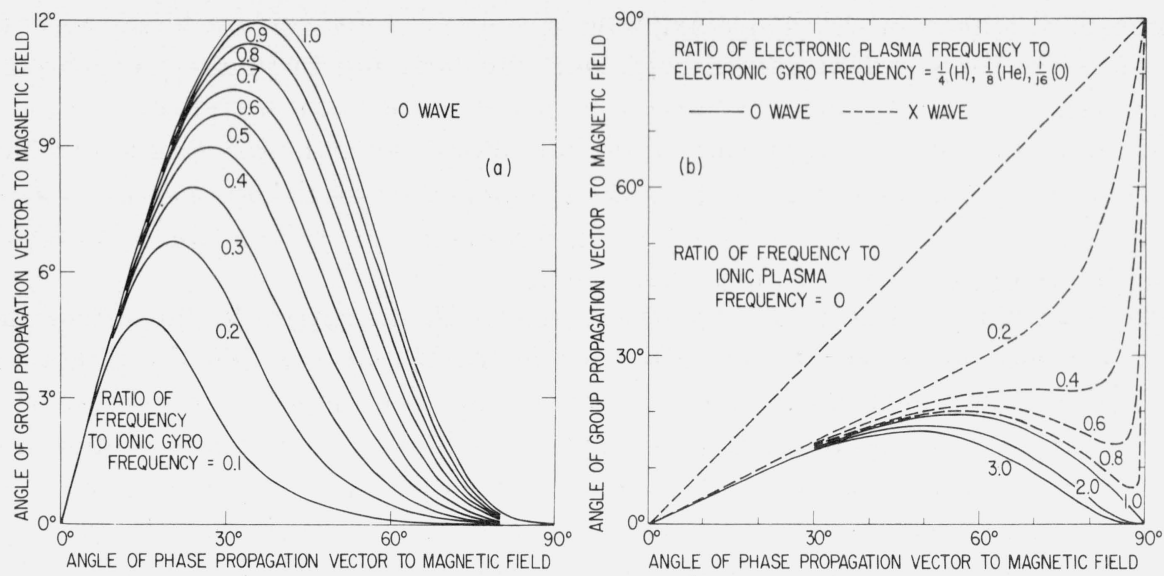

FIGURE 10. Illustrating the relation between the direction of group propagation and the direction of phase propagation (a) for the $L$ wave and (b) for the $R$ wave.
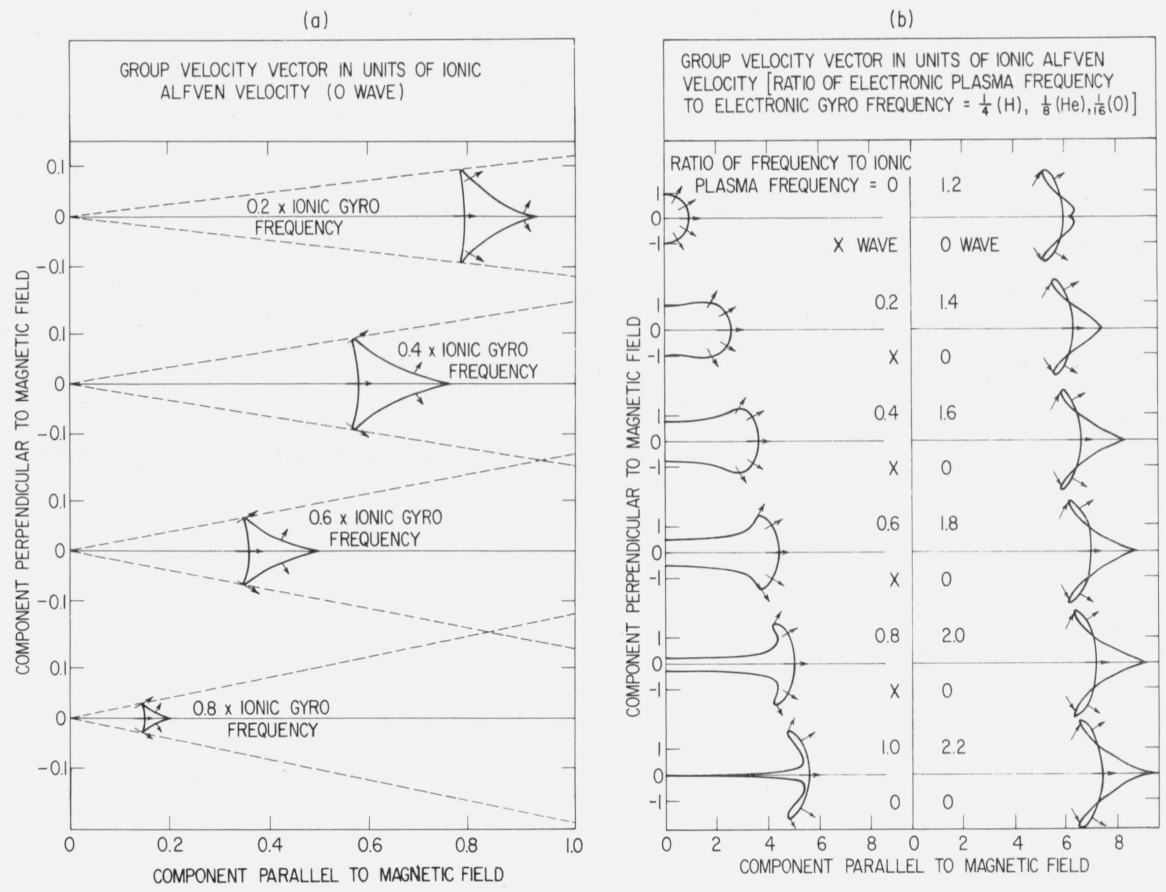

FIGURE 11. Illustrating the directional pulse patterns for a source at the origin (a) for the $L$ wave and (b) for the $R$ wave.

of times may be imagined by expanding the scale of the diagrams in figure 11 proportionally to elapsed time.

From figure 1la we see that, for the $\mathrm{L}$ wave in its hydromagnetic pass-band, a single pulse leaving the source gives rise to two pulses in the radiation field. Moreover, radiation is confined to a cone round the imposed magnetic field. The semi-angle of the cone decreases with frequency and is given approximately by

$$
0.28\left(\frac{\omega}{\omega_{M i}}\right)^{1 / 2}
$$


Thus a source of $\mathrm{L}$ waves in the hydromagnetic pass-band constitutes a beam antenna looking both ways along the imposed magnetic field, and the beaming becomes more pronounced at lower frequencies. As the frequency tends to zero, the pulse pattern shrinks to a pair of points moving away from the source in opposite directions along the imposed magnetic field with the ionic Alfvén velocity. On the other hand, as the frequency tends to the ionic gyrofrequency, the group velocity tends to zero. Above the ionic gyrofrequency we are in a stop-band for the $\mathrm{L}$ wave until a critical frequency is reached at radio frequencies.

The arrows in figure 11 indicate, in accordance with the angle $\theta_{p}$, the direction in which individual wave crests move within the pulse. In figure lla we see that the direction of phase propagation varies from longitudinal at the center of the slow pulse to transverse at the tip of the fast pulse. At this point group propagation is longitudinal although phase propagation is transverse. The variation in the magnitude of the phase velocity along the pulse pattern may be seen from the dependence of refractive index upon $\theta_{p}$ in figure 7 . With the aid of figure 10, this may be redrawn to show the variation of refractive index with $\theta_{g}$ as shown in figure 12 . At the tip of the fast pulse in figure 1la the phase velocity is zero in accordance with the infinity of refractive index in figure 12a.

Figure $11 \mathrm{~b}$ shows the directional pulse patterns for the $\mathrm{R}$ wave. At frequencies small compared with the ionic gyrofrequency this wave is radiated omnidirectionally with the ionic Alfvén velocity. As the frequency increases, the group velocity along the imposed magnetic field increases while that perpendicular to the imposed magnetic field decreases. At the bottom of the left half of figure $11 \mathrm{~b}\left(f=f_{\infty}\right)$ the group velocity perpendicular to the imposed field has been reduced to zero. At still higher frequencies $\left(f>f_{\infty}\right)$ radiation is confined to a cone round the imposed magnetic field as shown in the right half of figure $11 \mathrm{~b}$. Here we again have at the tip of the fast pulse a situation in which group propagation is longitudinal while phase propagation is transverse, the phase velocity being zero in accordance with the infinity of refractive index in figure $12 \mathrm{~b}$.

Figure 13 shows for group propagation how the hydromagnetic approximation fits onto the radio approximation. The left half of figure 13 contains a selection of the directional pulse patterns shown in figure $11 \mathrm{~b}$ and is based on the phase propagation diagram in figure 9a. The directional pulse patterns in the right half of figure 13 are based on the phase propagation diagram in figure $9 \mathrm{~b}$ and therefore on (56). In figure 13 it should be remembered that, in accordance with (53),
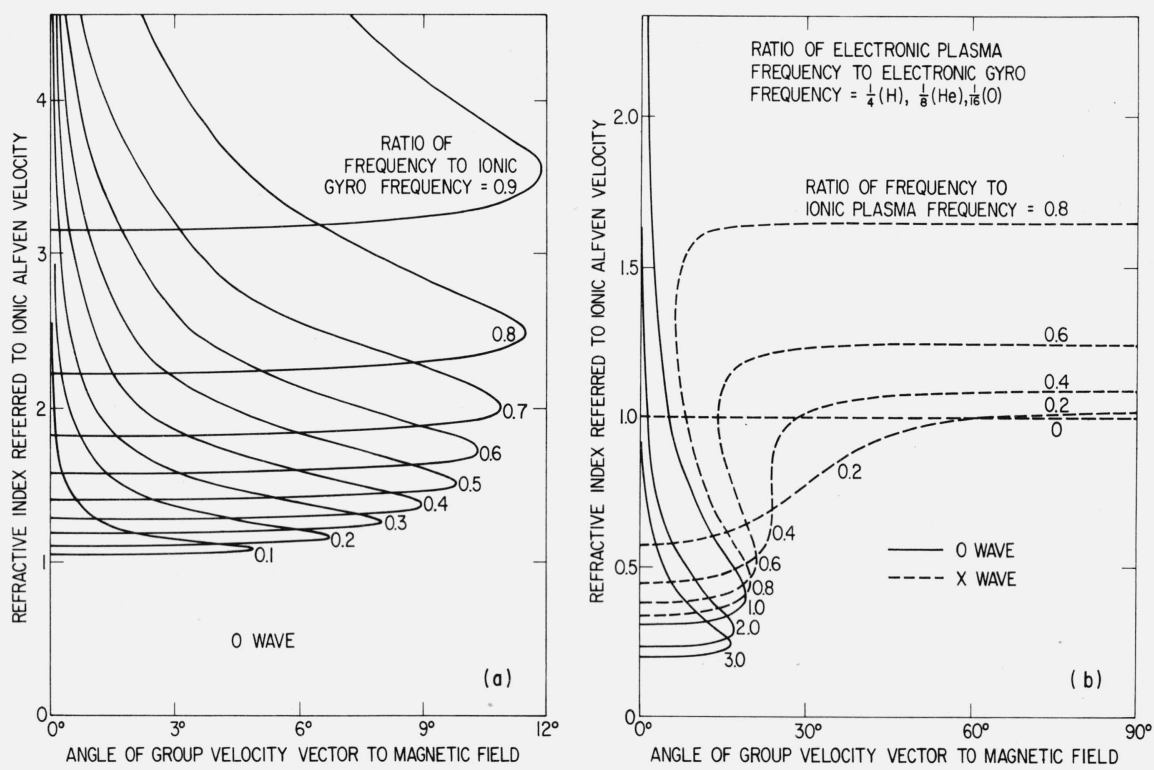

FIGURE 12. Illustrating the variation of refractive index with direction of group propagation (a) for the $L$ wave and (b) for the $R$ wave. 
FIGURE 13. Illustrating for the $R$ wave the fit between the directional pulse patterns derived from (a) the hydromagnetic approximation and (b) the radio approximation.

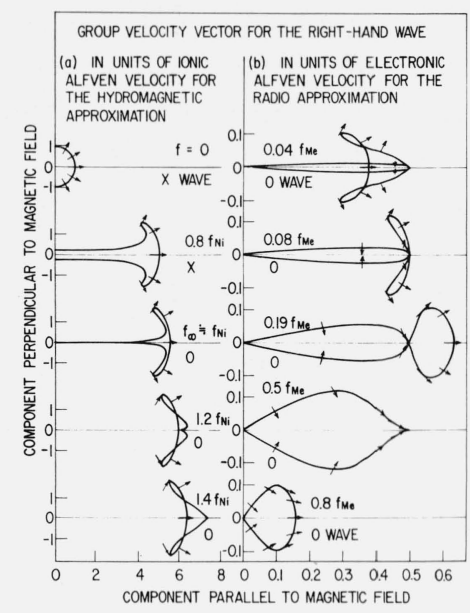

each wave packet moves radially away from the origin. Thus the portions of the pulse that trail back to or near the origin are in fact being stretched sideways and are therefore subject to more than inverse-square-law divergence. These trailing portions of the pulse are not of great practical importance. The trailing portion appearing in the top diagram on the right of figure 13 is $_{\star}$ completely omitted for the hydromagnetic approximation in the bottom diagram on the left. From figure 13 we obtain an integrated picture of how the omnidirectional magnetohydrodynamic wave at frequencies small compared with the ionic gyrofrequency becomes transformed into the phenomena encountered at whistler frequencies.

The following points should be noted about figure 13:

(1) Strongest beaming of the $\mathrm{R}$ wave along the imposed magnetic field occurs in the radio range. As shown by R. L. Smith [1960] the frequency for strongest beaming is approximately $0.19 f_{M e}$ and the corresponding half-angle of the cone is about $11^{\circ}$.

(2) In the frequency range $f_{\infty}<f<1 / 2 f_{M e}$ there is radiated along the imposed magnetic field a pulse for which the direction of phase propagation makes a large angle with the direction of the imposed field. As shown by Gendrin [1960], the group velocity for this pulse in the frequency range $f_{\infty} \ll f<1 / 2 f_{M e}$ is independent of frequency and is equal to one half the electronic Alfvén velocity.

(3) While the transition from omnidirectional propagation to beaming is shown in figure 13 as occurring near the ionic plasma frequency, in reality it occurs at the frequency $f_{\infty}$ given by (43) for the hydromagnetic approximation and by (45) when an approximate allowance is made for the effect of electron inertia at the ionic plasma frequency.

(4) Because of the effect of divergence in excess of that corresponding to the inverse square law, de facto beaming of the radiation occurs below the frequency $f_{\infty}$. This is true a fortiori when the effect of collisions is taken into account (see sec. 7). Beaming of the $\mathrm{R}$ wave along the imposed magnetic field is therefore significant for all frequencies large compared with the ionic gyrofrequency and appreciably less than the electronic gyrofrequency.

It should further be noted that the beaming indicated in figures 11 and 13 arises from the behavior of the magnetoplasma rather than from the characteristics of the source. It would also be possible for the source itself to have beaming properties. We may conveniently think of such a source as a broadside antenna that emphasizes a particular part of an angular spectrum of plane waves. To see which portion of a pulse pattern illustrated in figures 11 and 13 is emphasized by beaming of the source, we must look at the directions of phase propagation indicated by the arrows. To emphasize a particular portion of a pulse pattern by means of a broadside antenna, the plane of the antenna must be at right angles to the corresponding arrow in figures 11 and 13 . Thus to emphasize the central section of the slow pulse in figure 1la we would orient the plane of the broad- 
side antenna at right angles to the magnetic axis in the usual way. On the other hand, if we turned the plane of the antenna through a right angle, we would then emphasize the tip of the fast pulse; energy would slide off the face of the antenna sideways in the direction of the imposed magnetic field and in the reverse direction.

\section{Elliptical Polarization Phenomena}

The dispersion relation (21) was obtained by eliminating the quantities (20) from a set of linear equations relating them. We can now return to these linear equations and study the ratio of any two of the quantities (20). In particular we can study the ratio $E_{y} / E_{x}$, which describes the elliptical polarization of the electric vector in a magnetoionic wave. We have seen that, because of neglect of electron inertia in the hydromagnetic approximation, the electric vector vibrates in a plane perpendicular to the imposed magnetic field. Hence the ratio $E_{y} / E_{x}$ gives the complete information concerning the elliptical polarization of the electric vector for a magnetionic wave.

Let us suppose that the linear equations relating the quantities (20) are handled in such a way that all these quantities other than the ratio $E_{y} / E_{x}$ are eliminated. We are then left with the equations

$$
\left(\frac{k c}{\omega}\right)^{2}=1+\frac{\omega_{N i}^{2}}{\omega_{M i}^{2}} \frac{1-j \frac{\omega}{\omega_{M i}} \frac{E_{y}}{E_{x}}}{1-\frac{\omega^{2}}{\omega_{M i}^{2}}}
$$

and

$$
\left(\frac{k c}{\omega}\right)^{2} \cos ^{2} \theta_{p}=1+\frac{\omega_{N i}^{2}}{\omega_{M i}^{2}} \frac{1+j \frac{\omega}{\omega_{M i}} \frac{E_{x}}{E_{y}}}{1-\frac{\omega^{2}}{\omega_{M i}}} .
$$

It is the final elimination of $E_{y} / E_{x}$ between (63) and (64) that leads to the dispersion relation (21) and hence to (22) for the square of refractive index. If we substitute for the square of refractive index from (22) into (63) we obtain for the polarization ratio

$-j \frac{E_{y}}{E_{x}}=\frac{1}{\cos ^{2} \theta_{p}} \frac{\omega_{M i}}{\omega}\left[\frac{1}{2} \sin ^{2} \theta_{p}\left(1+\frac{\omega_{M i}^{2}}{\omega_{N i}^{2}}-\frac{\omega^{2}}{\omega_{N i}^{2}}\right) \pm \sqrt{1 / 4 \sin ^{4} \theta_{p}\left(1+\frac{\omega_{M i}^{2}}{\omega_{N i}^{2}}-\frac{\omega^{2}}{\omega_{N i}^{2}}\right)^{2}+\cos ^{2} \theta_{p} \frac{\omega^{2}}{\omega_{M i}^{2}}}\right]$.

Alternatively we may eliminate the square of refractive index between (63) and (64) and hence obtain a quadratic equation for the polarization ratios of the magnetoionic waves, namely

$$
\cos ^{2} \theta_{p} \frac{\omega}{\omega_{M i}}\left(-j \frac{E_{y}}{E_{x}}\right)^{2}-\sin ^{2} \theta_{p}\left(1+\frac{\omega_{M i}^{2}}{\omega_{N i}^{2}}-\frac{\omega^{2}}{\omega_{N i}^{2}}\right)\left(-j \frac{E_{y}}{E_{x}}\right)-\frac{\omega}{\omega_{M i}}=0
$$

The solution of this quadratic then gives (65).

The product of the roots of the quadratic (66) has a simple value from which we may derive the relation

$$
\left(\frac{E_{y} \cos \theta_{p}}{E_{x}}\right)_{0}\left(\frac{E_{y} \cos \theta_{p}}{E_{x}}\right)_{X}=1
$$

The product of the ratios $E_{y} \cos \theta_{p} / E_{x}$ for the two magnetoionic waves is equal to unity. This implies a simple relation between the electric ellipses for the two waves that may be described as 
follows. The two electric ellipses are in a plane perpendicular to the direction of the imposed field, and these ellipses may be orthogonally projected onto a plane of constant phase. Either of these projected ellipses may be obtained from the other by reversing the direction of rotation and turning the ellipse round the direction of phase propagation through an angle $1 / 2 \pi$. This relation between the electric ellipses of the two magnetoionic waves is the same as that familiar at radio frequencies [Booker, 1934].

The fact that the expression on the right-hand side of (65) is real implies that the components of electric field in and perpendicular to the $\left(\mathbf{k}, \mathbf{B}_{0}\right)$ plane vibrate in quadrature. The electric ellipse therefore lies in a plane perpendicular to $\mathbf{B}_{0}$ and has its principal axes in and perpendicular to the (k, $\mathbf{B}$ ) plane (see fig. 1). The expression on the right-hand side of (65) is the ratio of the axis in the $\left(\mathbf{k}, \mathbf{B}_{0}\right)$ plane to the axis perpendicular to this plane. Positive values imply left-handed polarization about $\mathbf{B}_{0}$ and negative values right-handed polarization. The values \pm 1 correspond to circular polarization and the values $0, \infty$ to linear polarization. The polarization ratio $-j E_{y} / E_{x}$ is shown in figure 14 as a function of frequency for a series of values of $\theta_{p}$. We notice that the polarization ratios for the $\mathrm{L}$ and $\mathrm{R}$ waves have opposite signs corresponding to left and right handed rotation about $\mathbf{B}_{0}$ regardless of the direction of propagation.

From figure 14b we see that the electric ellipse for the $\mathrm{R}$ wave at the ionic gyrofrequency is a circle perpendicular to the imposed magnetic field regardless of the direction of propagation. On the other hand, we see from figure 14a that, for nearly longitudinal propagation of the $\mathrm{L}$ wave at frequencies small compared with the ionic gyrofrequency, polarization varies rapidly from circular to linear. In fact, interchange in the order of the limiting processes $\theta_{p} \rightarrow 0$ and $f \rightarrow 0$ effects a switch from linear to circular polarization. This is because the curve indicating transition from quasi-transverse to quasi-longitudinal propagation passes through the origin in figure $5 \mathrm{a}$. The

FIGURE 14. Illustrating elliptical polarization properties of the electric vector, the magnetic vector and the electronic velocity (a) for the $L$ wave and (b) for the $R$ wave.
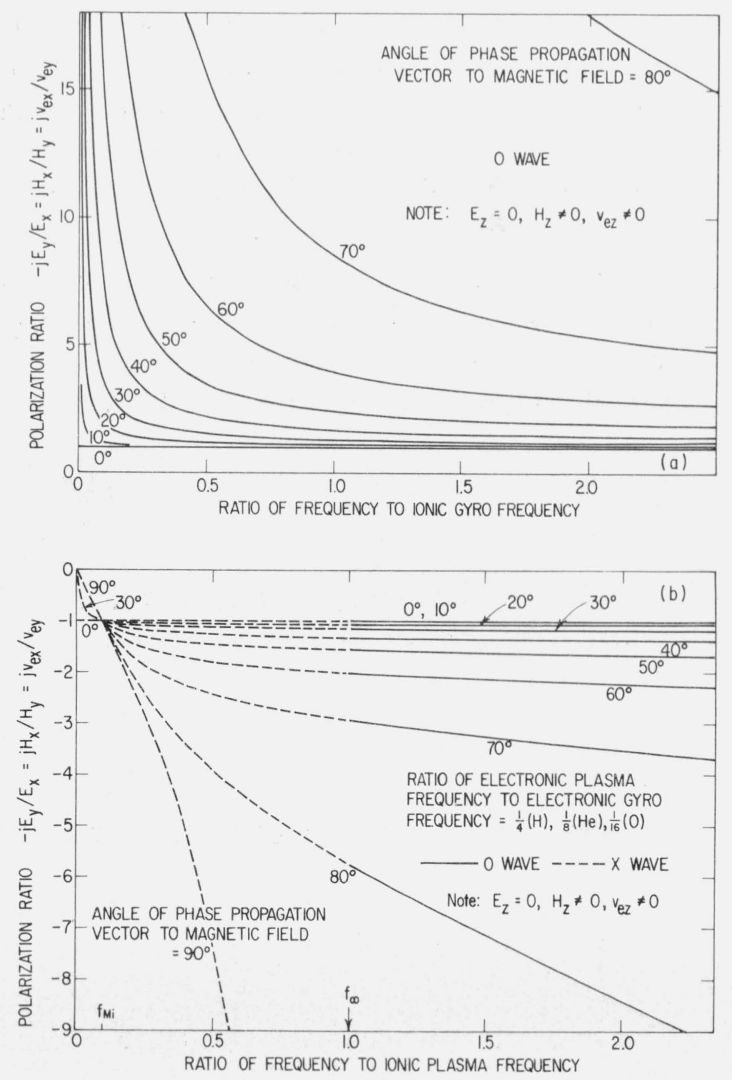
behavior of this curve near the origin is given by

$$
\theta_{p}^{2}=2 \frac{1}{1+\frac{\omega_{M i}^{2}}{\omega_{N i}^{2}}} \frac{\omega}{\omega_{M i}}
$$

from which we see that, when $\theta_{p}$ and $\omega / \omega_{M i}$ are both small, the elliptical polarization of the $\mathrm{L}$ wave is vitally dependent on the ratio of $\theta_{p}^{2}$ to $\omega / \omega_{M i}$.

Using figure 10 , the angle $\theta_{p}$ between the direction of phase propagation and the direction of the imposed magnetic field may be converted into the angle $\theta_{g}$ between the direction of group propagation and the direction of the imposed magnetic field. In this way figure 14, as well as a number of ensuing diagrams, may be replotted in terms of $\theta_{g}$. While the resulting curves are of interest, they are mostly omitted to conserve space. As an alternative, we may remember that $\theta_{p}$ varies from 0 to $1 / 2 \pi$ as we move from the center of the slow pulse to the tip of the fast pulse both in figure $11 \mathrm{a}$ and in the right half of figure $11 \mathrm{~b}$ as shown by the arrows. It follows from figure 14 that the electric vector is circularly polarized in a plane perpendicular to the magnetic axis at the center of the slow pulse, but changes via an elliptical vibration to an almost linear vibration radial to the magnetic axis at the tip of the fast pulse. At the tip $\mathbf{E}$ vanishes for the hydromagnetic approximation although there is some vibration of $\mathbf{E}$ along the magnetic axis when electronic inertia is taken into account.

Turning now to the elliptical polarization of the magnetic vector in a magnetoionic wave, we remember that, in accordance with (15), this vector vibrates in a plane perpendicular to the direction of phase propagation. Nevertheless, the magnetic ellipse is simply related to the electric ellipse. From the $x$ and $y$ components of (15) it follows that

$$
\frac{H_{x}}{H_{y}}=-\frac{E_{y}}{E_{x}} .
$$

This equation implies that the orthogonal projection of the magnetic ellipse onto the plane of the electric ellipse is identical in shape with the electric ellipse except for rotation through $1 / 2 \pi$ round the direction of the imposed magnetic field. Figure 14 may also be interpreted therefore to give the elliptical polarization of the magnetic field of a magnetoionic wave.

The electronic ellipses of the magnetoionic waves may be studied by using the linear equations relating the quantities (20) to obtain the ratios of $v_{e x}, v_{e y}$ and $v_{e z}$. It turns out that the plane of the electronic ellipse does not in general coincide with either the plane of the electric ellipse or the plane of the magnetic ellipse. However the projection of the electronic ellipse onto a plane perpendicular to the imposed magnetic field is identical in shape and orientation to the projection of the magnetic ellipse. Hence the left hand side of (65) may be replaced by

$$
-j \frac{E_{y}}{E_{x}}=j \frac{H_{x}}{H_{y}}=j \frac{v_{e x}}{v_{e y}}
$$

and figure 14 may be interpreted accordingly. However the normal to the electronic ellipse is turned away from the vector $\mathbf{B}_{0}$ towards the vector $\mathbf{k}$ through the angle

$$
\theta_{e}=-\tan ^{-1} \frac{v_{e z}}{v_{e y}}
$$

and it follows from the linear equations relating the quantitites (20) that

$$
\frac{v_{e z}}{v_{e y}}=\cos \theta_{p} \sin \theta_{p} \frac{\omega \omega_{M i}}{\omega_{N i}^{2}}\left(\frac{k c}{\omega}\right)^{2}\left(-j \frac{E_{y}}{E_{x}}\right)
$$

Using (71) and (72) we may convert figures 7 and 14 into figure 15. 

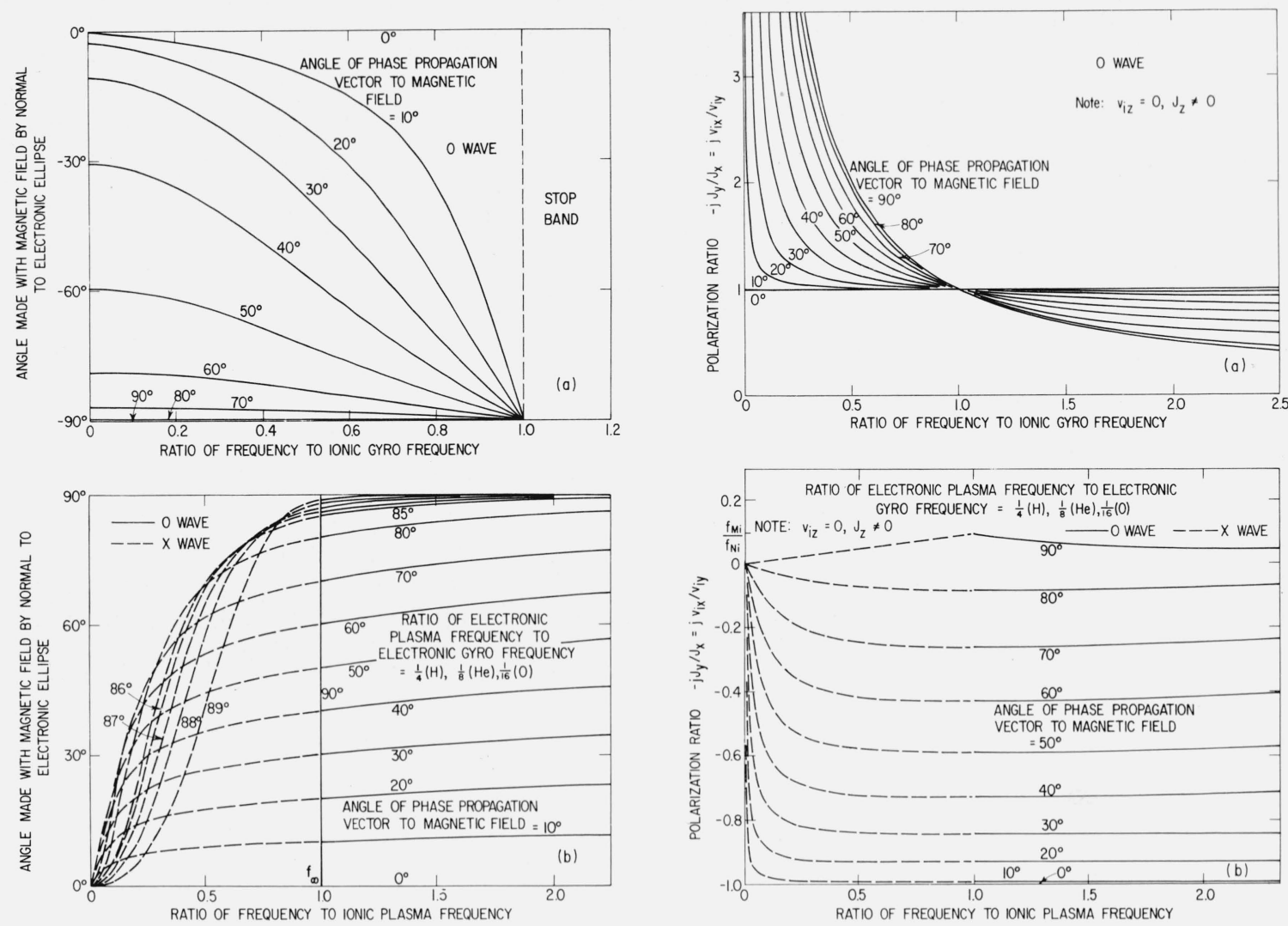

FIGURE 15. Illustrating variation of the orientation of the plane of the electronic ellipse (a) for the $L$ wave and (b) for the $R$ wave.

FIGURE 16. Illustrating elliptical polarization properties of the electric current vector and the ionic velocity $(a)$ for the $L$ wave and (b) for the $R$ wave.

For the $\mathrm{L}$ wave in its hydromagnetic pass-band figure 15 a shows that the values of $\theta_{e}$ are negative. This implies that, as the direction of phase propagation turns away from the direction of the imposed magnetic field, the normal to the electronic ellipse turns in the opposite direction. However, these are circumstances in which great importance attaches to the motion of the ions even from the viewpoint of electric current.

For the $\mathrm{R}$ wave we have positive ordinates in figure $15 \mathrm{~b}$, showing that the normal to the electronic ellipse turns away from $\mathbf{B}_{0}$ in the same direction as $\mathbf{k}$. Moreover, for $f>f_{\infty}$, the electronic ellipse does not depart radically from perpendicularity to the direction of phase propagation. In the right half of figure $11 \mathrm{~b}\left(f>f_{\infty}\right)$ the electronic motion is circularly polarized in a plane perpendicular to the magnetic axis at the center of the slow pulse, but changes via an elliptical motion to an almost rotary vibration round the magnetic axis near the tip of the fast pulse. At the tip the electronic motion vanishes for the hydromagnetic approximation, although there is some motion along the magnetic axis when electronic inertia is taken into account. On the other hand, for $f<f_{\infty}$, figure $15 \mathrm{~b}$ shows that, for the $\mathrm{R}$ wave, $\theta_{e}$ at first increases from zero with $\theta_{p}$ and then decreases again to zero as $\theta_{p}$ increases further to $1 / 2 \pi$. Thus, in the left half of figure $11 \mathrm{~b}\left(f<f_{\infty}\right)$, the plane of the electronic ellipse is perpendicular to the magnetic axis where the pulse pattern crosses this axis, begins to turn the same way as the direction of phase propagation as we move round the pulse pattern, but returns to a position normal to the imposed magnetic field where the pulse pattern crosses the perpendicular axis. For frequencies small compared with the ionic gyrofrequency, the plane of the electronic ellipse for the $R$ wave does not depart much from perpendicularity to the magnetic axis; the electronic motion varies from circular polarization where the pulse pattern crosses the magnetic axis to almost linear vibration at right angles to $\mathbf{B}_{0}$ in the $\left(\mathbf{k}, \mathbf{B}_{0}\right)$ plane where the pulse pattern crosses the perpendicular axis. 
For (65) the quasi-longitudinal and quasi-transverse approximations are

$$
\begin{aligned}
& -j \frac{E_{y}}{E_{x}}=j \frac{H_{x}}{H_{y}}=j \frac{v_{e x}}{v_{e y}}= \pm \frac{1}{\cos \theta_{p}} \\
& -j \frac{E_{y}}{E_{x}}=j \frac{H_{x}}{H_{y}}=j \frac{v_{e x}}{v_{e y}}=\frac{\omega_{M i}}{\omega}\left(1+\frac{\omega_{M i}^{2}}{\omega_{N i}^{2}}-\frac{\omega^{2}}{\omega_{N i}^{2}}\right) \tan ^{2} \theta_{p} \quad(\mathrm{QT}, \mathrm{O}) \\
& -j \frac{E_{y}}{E_{x}}=j \frac{H_{x}}{H_{y}}=j \frac{v_{e x}}{v_{e y}}=-\frac{\omega_{M i}}{1+\frac{\omega_{M i}^{2}}{\omega_{N i}^{2}}-\frac{\omega^{2}}{\omega_{N i}^{2}}} \frac{1}{\sin ^{2} \theta_{p}} \quad(\mathrm{QT}, \mathrm{X}) \cdot
\end{aligned}
$$

A number of features of figure 14 may be traced with the aid of these approximations.

For the hydromagnetic approximation, the ionic ellipses for the magnetoionic waves lie in planes perpendicular to the imposed magnetic field because, as already mentioned in section 2 , there is no vibration of ions parallel to the imposed magnetic field, there being no electric field in this direction to drive them. On the other hand, the ionic ellipses are not in general identical in shape with any of the ellipses so far discussed. From the linear equations relating the quantities (20) it follows that

$$
j \frac{v_{i x}}{v_{i y}}=\frac{\frac{\omega}{\omega_{M i}}+\left(-j \frac{E_{y}}{E_{x}}\right)}{1+\frac{\omega}{\omega_{M i}}\left(-j \frac{E_{y}}{E_{x}}\right)} .
$$

With the aid of this equation curves for the polarization ratio for the ions may be deduced from figure 14 giving the results shown in figure 16 .

From figure 16a we see that, for the $\mathrm{L}$ wave at the ionic gyrofrequency, the ions are circularly polarized in a plane perpendicular to the imposed magnetic field regardless of the direction of propagation. We are here at the high frequency end of the hydromagnetic pass-band for the $\mathrm{L}$ wave where the wave is known as the ion cyclotron wave. At the center of the slow pulse in figure 11a the ionic polarization is circular. On the other hand, at the tip of the fast pulse, the ionic motion involves an oscillation around the magnetic axis combined with a quadrature oscillation radial to the magnetic axis.

From figure $16 \mathrm{~b}$ we see that, for the $\mathrm{R}$ wave, the sign of the ionic polarization ratio for trans. verse propagation is not the same as for longitudinal propagation. This means that, when the direction of phase propagation in the $\mathrm{R}$ wave is sufficiently transverse to the imposed magnetic field, the ions execute ellipses in planes perpendicular to the imposed magnetic field in the lefthand sense. Thus, whereas for the hydromagnetic approximation the magnetoionic waves may be classified into left-handed and right-handed waves from the viewpoint of electromagnetic theory, the same is not true from the viewpoint of fluid mechanics. However, when the direction of rotation of the ions for the $\mathrm{R}$ wave is reversed, they are normally executing quite narrow ellipses, and the ionic motion is almost a linear vibration perpendicular to $\mathbf{B}_{0}$ in the $\left(\mathbf{k}, \mathbf{B}_{0}\right)$ plane. In the left half of figure 1lb $\left(f<f_{\infty}\right)$ the fluid motion varies from circularly polarized near the magnetic axis to almost linear vibration in the direction of propagation near the perpendicular axis. At the tip of the fast pulse on the right half of figure $11 \mathrm{~b}\left(f>f_{\infty}\right)$ the fluid vibration is mainly radial to the magnetic axis, although there is some quadrature rotary vibration round the magnetic axis.

The electric current density $\mathbf{J}$ is derivable from the ionic and electronic vibrations in accordance with (6). Since the plane of the electronic ellipse is tilted with respect to the plane of the ionic ellipse, the same is true for the current ellipse. However, it emerges that

$$
\frac{J_{y}}{J_{x}}=-\frac{v_{i x}}{v_{i y}}
$$


FIGURE 17. Illustrating variation of the orientation of the plane of the current ellipse for the $R$ wave.

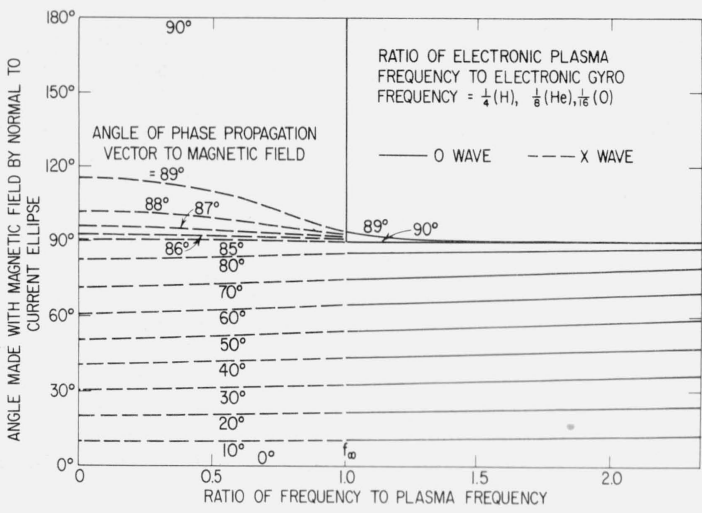

so that the orthogonal projection of the current ellipse onto a plane perpendicular to the imposed magnetic field is identical with the ionic ellipse except for rotation through a right angle. Thus figure 16 may also be interpreted as giving the projection of the current ellipse onto a plane perpendicular to the imposed magnetic field. However, the normal to the current ellipse in general makes with the direction of the imposed magnetic field a nonzero angle $\theta_{J}$ measured from $\mathbf{B}_{0}$ towards $\mathbf{k}$ and given by

$$
\theta_{J}=-\tan ^{-1} \frac{J_{z}}{J_{y}}
$$

From (6), together with the linear equations relating the quantities (20), we may deduce that

$$
\tan \theta_{J}=\tan \theta_{p}\left[1+\frac{\omega_{M i}^{2}}{\omega_{N i}^{2}} \frac{\left(1-\frac{\omega^{2}}{\omega_{M i}^{2}}\right)\left(-j \frac{E_{y}}{E_{x}}\right)}{\frac{\omega}{\omega_{M i}}+\left(-j \frac{E_{y}}{E_{x}}\right)}\right] .
$$

Using this equation we may calculate $\theta_{J}$ from the information plotted in figure 14 . However, for the $\mathrm{L}$ wave in its hydromagnetic pass-band, the quantity in square brackets in (79) is practically unity if $\omega_{M i} \ll \omega_{N i}$, so that the current ellipse is almost exactly perpendicular to the direction of phase propagation. For the $\mathrm{R}$ wave, however, departures from this occur as shown in figure 17. These departures are particularly noticeable for $f<f_{\infty}$ and $\theta_{p}>\tan ^{-1} f_{N i} / f_{M i}$. For $f<f_{\infty}$ the angle $\theta_{J}$ increases from 0 to about $1 / 2 \pi$ as $\theta_{p}$ increases from 0 to about $\tan ^{-1} f_{N i} / f_{M i}$, but then increases further to $\pi$ as $\theta_{p}$ increases to $1 / 2 \pi$. During the latter stage, therefore, the current vector rotates left-handed about the direction of the imposed magnetic field even though we are discussing the $\mathrm{R}$ wave. This is a consequence of the corresponding behavior of the ionic ellipse. Thus, in the left half of figure $11 \mathrm{~b}\left(f<f_{\infty}\right)$, the current ellipse is perpendicular to the direction of the imposed magnetic field both where the pulse pattern crosses the magnetic axis and where it crosses the perpendicular axis, and the plane of the ellipse makes a complete half revolution between the two positions. Moreover, we see from figure 17 that this is true even in the limit of zero frequency.

A number of features of figure 16 may be seen by means of the quasi-longitudinal and the quasitransverse approximations obtained by substituting from (73), (74), and (75) into (76). We obtain

$$
-j \frac{J_{y}}{J_{x}}=j \frac{v_{i x}}{v_{i y}}=\frac{\frac{\omega}{\omega_{M i}} \pm \frac{1}{\cos \theta_{p}}}{1 \pm \frac{\omega}{\omega_{M i}} \frac{1}{\cos \theta_{p}}}
$$




$$
\begin{aligned}
& -j \frac{J_{y}}{J_{x}}=j \frac{v_{i x}}{v_{i y}}=\frac{\omega_{M i}}{\omega} \frac{\frac{\omega^{2}}{\omega_{M i}^{2}}+\left(1+\frac{\omega_{M i}^{2}}{\omega_{N i}^{2}}-\frac{\omega^{2}}{\omega_{N i}^{2}}\right) \tan ^{2} \theta_{p}}{1+\left(1+\frac{\omega_{M i}^{2}}{\omega_{N i}^{2}}-\frac{\omega^{2}}{\omega_{N i}^{2}}\right) \tan ^{2} \theta_{p}} \quad(\mathrm{QT}, \mathrm{O}) \\
& -j \frac{J_{y}}{J_{x}}=j \frac{v_{i x}}{v_{i y}}=\frac{\omega}{\omega_{M i}} \frac{1-\left(1+\frac{\omega_{M i}^{2}}{\omega_{N i}^{2}}-\frac{\omega^{2}}{\omega_{N i}^{2}}\right) \sin ^{2} \theta_{p}}{\frac{\omega^{2}}{\omega_{M i}^{2}}-\left(1+\frac{\omega_{M i}^{2}}{\omega_{N i}^{2}}-\frac{\omega^{2}}{\omega_{N i}^{2}}\right) \sin ^{2} \theta_{p}} \quad(\mathrm{QT}, \mathrm{X}) .
\end{aligned}
$$

For strictly transverse propagation, (81) and (82) become

$$
\begin{array}{ll}
-j \frac{J_{y}}{J_{x}}=j \frac{v_{i x}}{v_{i y}}=\frac{\omega_{M i}}{\omega} & (\mathrm{T}, \mathrm{O}) \\
-j \frac{J_{y}}{J_{x}}=j \frac{v_{i x}}{v_{i y}}=\frac{\omega \omega_{M i}}{\omega_{N i}^{2}} \frac{1}{1+\frac{\omega_{M i}^{2}}{\omega_{N i}^{2}}} & (\mathrm{~T}, \mathrm{X})
\end{array}
$$

and from these equations the behavior of the $90^{\circ}$ curve in figure $16 \mathrm{~b}$ may be verified, thus confirming in these circumstances the left-handed behavior of the $\mathrm{R}$ wave so far as electric current and fluid motion are concerned.

\section{Comparison of Ionic and Electronic Motions}

To compare the ionic and electronic motions in a magnetoionic wave it is appropriate not merely to compare their elliptical polarizations, but also to compare the actual amplitudes of vibration. From the linear equations relating the quantities (20), we may derive expressions for the ratio of electronic velocity to ionic velocity (a) for the component of motion perpendicular to the (k, $\left.\mathbf{B}_{0}\right)$ plane and (b) for the component of motion in the $\left(\mathbf{k}, \mathbf{B}_{0}\right)$ plane perpendicular to $\mathbf{B}_{0}$. We obtain

$$
\frac{v_{e x}}{v_{i x}}=1-\frac{\omega / \omega_{M i}}{j v_{i x} / v_{i y}}
$$

and

$$
\frac{v_{e y}}{v_{i y}}=1-\frac{\omega}{\omega_{M i}}\left(j \frac{v_{i x}}{v_{i y}}\right)
$$

For the component along the imposed magnetic field the ionic motion is zero for the hydromagnetic approximation. By means of (85) and (86) we may convert figure 16a into figure 18.

We notice that, for longitudinal propagation of the $\mathrm{L}$ wave at frequencies small compared with the ionic gyrofrequency, the ionic and electronic motions are almost indetical so that the corresponding contributions to the electric current almost cancel. It should be noted, however, that it is the existence of the "small" difference current that causes the change in the velocity of propagation from the free space value to the Alfvén value, and this change is normally far from "small."

For the $\mathrm{L}$ wave at the ionic gyrofrequency, there is no motion of the electrons in a plane perpendicular to the imposed magnetic field regardless of the direction of propagation. However, it follows from figure 15a that there is motion of the electrons along $\mathbf{B}_{0}$ except when the direction of phase propagation is also along $\mathbf{B}_{\mathbf{0}}$. Thus, for the ion cyclotron wave, there is circular motion of the ions in planes perpendicular to the imposed magnetic field combined with linear vibration 


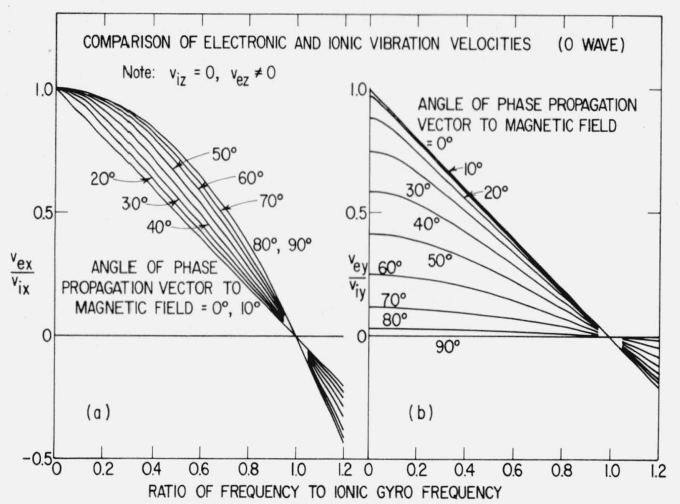

FIGURE 18. Illustrating for the $L$ wave variation of the ratio of electronic velocity to ionic velocity (a) for the component of motion perpendicular to the $\left(\mathbf{k}, \mathbf{B}_{0}\right)$ plane and (b) for the component of motion in the $\left(\mathbf{k}, \mathbf{B}_{0}\right)$ plane perpendicular to $\mathbf{B}_{0}$.

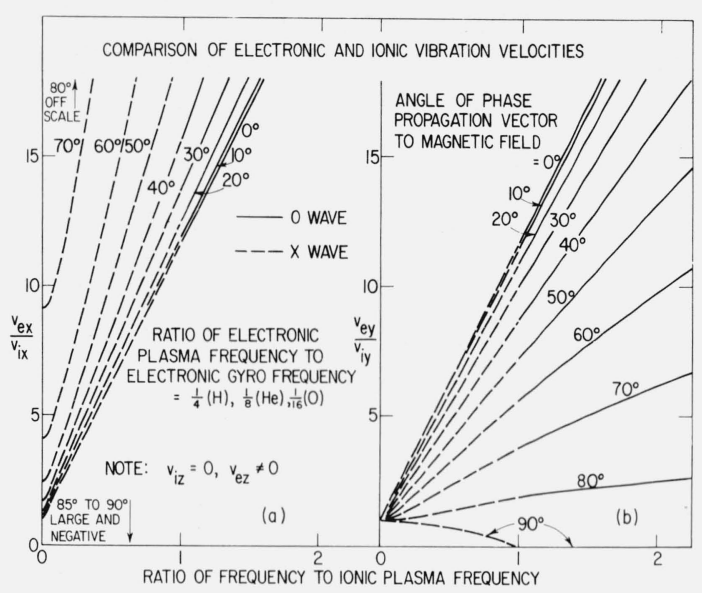

FiguRE 19. Illustrating for the $R$ wave variation of the ratio of electronic velocity to ionic velocity (a) for the component of motion perpendicular to the $\left(\mathbf{k}, \mathbf{B}_{0}\right)$ plane and (b) for the component of motion in the $\left(\mathbf{k}, \mathbf{B}_{0}\right)$ plane perpendicular to $\mathbf{B}_{0}$.

of electrons along the imposed magnetic field, the latter motion vanishing for strictly longitudinal propagation.

In figure 1la the ionic and electronic motions are circularly polarized and in phase at the center of the slow pulse, with the positive contribution of the ions to the electric current outbalancing the negative contribution of the electrons. As we move to the tip of the fast pulse, the ionic ellipse remains perpendicular to $\mathbf{B}_{0}$ whereas the electronic ellipse rotates in the opposite direction to $\mathbf{k}$ as shown in figure 15a. The combination produces a current ellipse that turns so as to be almost exactly perpendicular to the direction of phase propagation. At the tip of the fast pulse the electric current is dominated by oscillation of the electrons along the imposed magnetic field.

For the $\mathrm{R}$ wave we may use (85) and (86) to convert figure $16 \mathrm{~b}$ into figure 19 . We notice that, at frequencies large compared with the ionic gyrofrequency, the electronic motion far exceeds the ionic motion. This is what, at sufficient high frequencies, justifies use of the radio approximation. However, it should be noted from figure $19 \mathrm{~b}$ that $v_{e y}<v_{i y}$ when the direction of phase propagation is nearly transverse to the imposed magnetic field. In these circumstances, therefore, the negative contribution to the electric current from the small component of electronic motion in the $\left(\mathbf{k}, \mathbf{B}_{0}\right)$ plane perpendicular to $\mathbf{B}_{0}$ is outbalanced by the positive contribution from the ionic motion. This is why, for nearly transverse propagation, the electric current has lefthanded polarization for the $\mathrm{R}$ wave even though the electronic motion has right-handed polarization (compare fig. 16b with fig. 14b).

Equations (12) and (13) show that, except for propagation strictly along the imposed magnetic field, the magnetoionic waves involve compressions and rarifications in both electron density and ion density. In fact these equations state that the fractional increase in density is equal to the product of the refractive index and the component of particle velocity in the direction of phase propagation, the velocity being expressed as a ratio to the standard velocity used in specifying refractive index (either the free space velocity or the ionic Alfvén velocity). Consequently, if the ratio to the ionic Alfvén velocity of the particle velocity in the direction of phase propagation is known, we merely multiply this by the appropriate ordinate in figure 7 to obtain the fractional increment in particle density. Near the infinite ordinates in figure 7 the assumption of a linearized cold plasma breaks down.

It is also interesting to compare the fluctuations in electron density with those in ion density. From (12) and (13) we obtain

$$
\frac{\delta N_{e}}{\delta N_{i}}=\frac{v_{e y} \sin \theta_{p}+v_{e z} \cos \theta_{p}}{v_{i y} \sin \theta_{p}}
$$




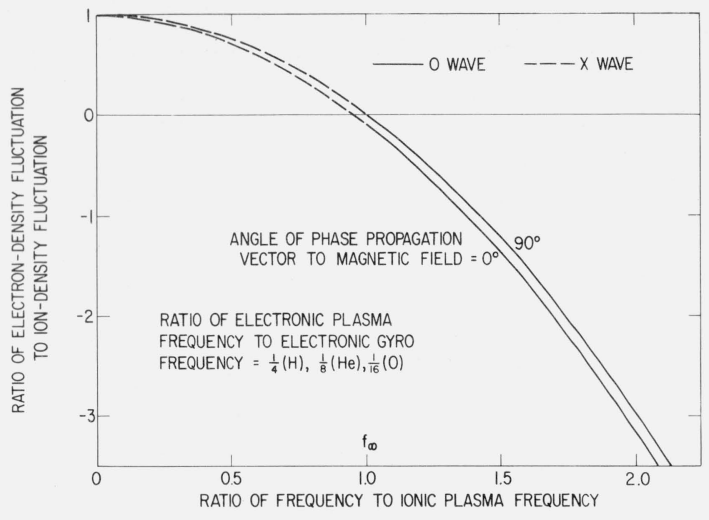

FIGURE 20. Illustrating the variation in the ratio of electron density to ion density for the $R$ wave.

and using (71), this may be rewritten

$$
\frac{\delta N_{e}}{\delta N_{i}}=\frac{v_{e y}}{v_{i y}}\left(1-\frac{\tan \theta_{e}}{\tan \theta_{p}}\right)
$$

We may therefore use the information plotted in figures $15,18 \mathrm{~b}$, and $19 \mathrm{~b}$ to derive $\delta N_{e} / \delta N_{i}$. For the $\mathrm{L}$ wave in its hydromagnetic pass-band this ratio is almost exactly unity independent of the direction of propagation. Except for strictly longitudinal propagation, there are fluctuations in both the ion density and the electron density but very little fluctuation in charge density. Except for frequencies small compared with the ionic gyrofrequency, the motions of the ions and electrons are different, but the components of motion perpendicular to the direction of phase propagation are almost exactly the same so that the net fluctuation in space-charge is practically zero. For the $\mathrm{R}$ wave, on the other hand, $\delta N_{e} / \delta N_{i}$ departs from unity for frequencies large compared with the ionic gyrofrequency. The dependence on direction of propagation is relatively slight as shown in figure 20 , but it should be remembered that both $\delta N_{e}$ and $\delta N_{i}$ vanish for strictly longitudinal propagation. Motion of the ions plays an essential role in the fluctuation of space-charge until the frequency is high compared with $f_{\infty}$.

\section{Ionic Attenuation}

It is well known that, for the radio approximation, it is easy to allow for electronic collisions by replacing the electronic mass $m_{e}$ by $m_{e} U_{e}$ where

$$
U_{e}=1-j \frac{\nu_{e}}{\omega}
$$

In the same way, for the hydromagnetic approximation, it is easy to allow for collisions of ions with neutral particles provided that the neutral gas is sufficiently massive to take no significant part in the wave motion. If $\nu_{i}$ is the ionic collisional frequency involved, we add the term $-m_{i} \nu_{i} \mathbf{v}_{i}$ to the right hand side of (2). In (11) this has the effect of replacing the ionic mass $m_{i}$ by $m_{i} U_{i}$ where

$$
U_{i}=1-j \frac{\nu_{i}}{\omega}
$$

Consequently, to allow for ionic collisions with a neutral gas that takes no significant part in the wave motion, we modify the dispersion relation (21), and all subsequent equations, by replacing

$$
\omega_{M i} \text { by } \omega_{M i} / U_{i}
$$




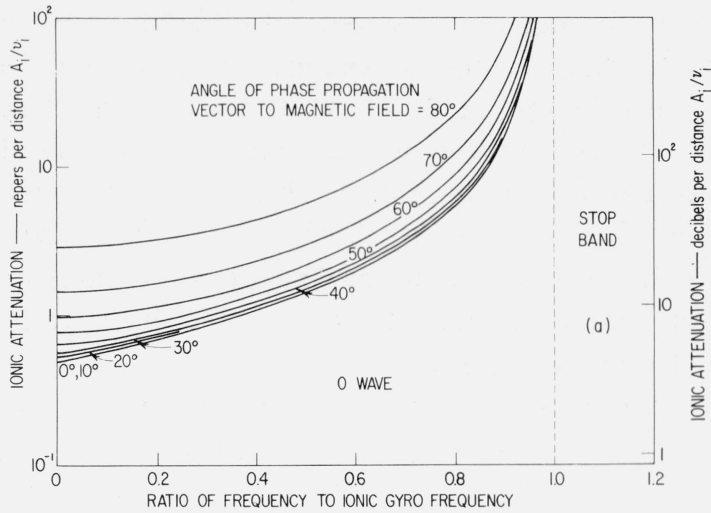

FiguRE 21. Illustrating the variation in the rate of ionic attenuation (a) for the $L$ wave and (b) for the $R$ wave.

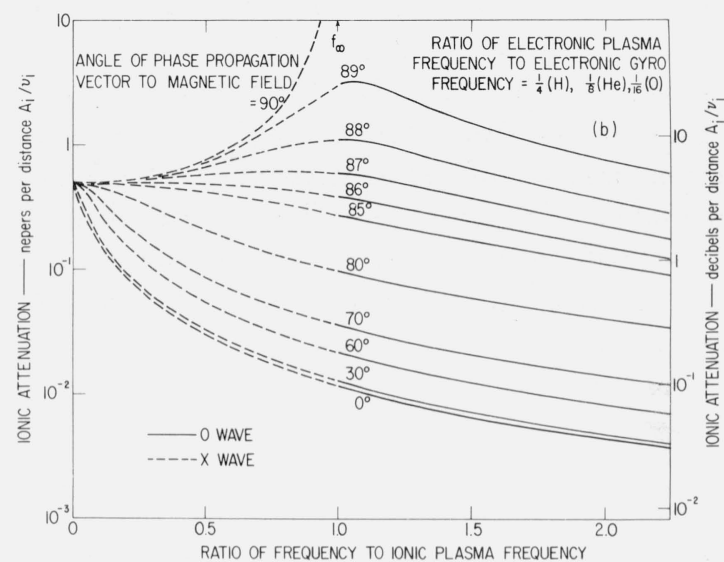

and

$$
\omega_{N i}^{2} \text { by } \omega_{N i}^{2} / U_{i} \text {. }
$$

To calculate the first order effect of ionic attentuation we may make the replacements (91) and (92) in the dispersion relation (55) and then expand to the first order in powers of $\left(U_{i}-1\right)$. We obtain in this way

$$
k=k_{U_{i}=1}-j \frac{\nu_{i}}{\omega}\left(\frac{\partial k}{\partial U_{i}}\right)_{U_{i}=1}
$$

so that the rate of attenuation in nepers per unit distance is

$$
\alpha=\frac{\nu_{i}}{\omega}\left(\frac{\partial k}{\partial U_{i}}\right)_{U_{i}=1}
$$

By differentiating the modified dispersion equation with respect to $U_{i}$ and then putting $U_{i}=1$, we derive

$$
\frac{\alpha A_{i}}{\nu_{i}}=\frac{\frac{\omega}{\omega_{M i}}\left[2 \cos ^{2} \theta_{p}\left(\frac{k c}{\omega_{N i}}\right)^{4}+\left(1+\cos ^{2} \theta_{p}\right)\left(1-2 \frac{\omega^{2}}{\omega_{N i}^{2}}\right)\left(\frac{k c}{\omega_{N i}}\right)^{2}-2\left(1+\frac{\omega_{M i}^{2}}{\omega_{N i}^{2}}-\frac{\omega^{2} \omega_{M i}^{2}}{\omega_{N i}^{2}}\right) \frac{\omega^{2}}{\omega_{M i}^{2}}\right]}{2 \frac{k c}{\omega_{N i}}\left[2 \cos ^{2} \theta_{p}\left(1-\frac{\omega^{2}}{\omega_{M i}^{2}}\right)\left(\frac{k c}{\omega_{N i}}\right)^{2}-\left(1+\cos ^{2} \theta_{p}\right)\left(1+\frac{\omega_{M i}^{2}}{\omega_{N i}^{2}}-\frac{\omega^{2}}{\omega_{N i}^{2}}\right) \frac{\omega^{2}}{\omega_{M i}^{2}}\right]} .
$$


This is the number of nepers of ionic attenuation suffered in the distance $A_{i} / \nu_{i}$, which is the wavelength obtained by converting the ionic collisional frequency into wavelength using the ionic Alfvén velocity. The rate of attenuation derived from (95) is illustrated in figure 21 . From figure 21 a we see that the attenuation of the $\mathrm{L}$ wave becomes large as the frequency increases to the ionic gyrofrequency, where the wave is the ion cyclotron wave. From figure $2 \mathrm{lb}$ we see that, for the $\mathrm{R}$ wave, the attenuation at low frequencies is half a neper per distance $A_{i} / \nu_{i}$ and decreases with increasing frequency except when the direction of phase propagation is nearly transverse to the imposed magnetic field. For the latter situation there is a maximum of attenuation near the frequency $f_{\infty}$ given by (43) for the hydromagnetic approximation and by (45) when electron inertia is taken approximately into account at the ionic plasma frequency. If attenuation is important, it is more important for propagation perpendicular to the magnetic axis than for propagation along it. Thus the effect of attenuation in the left half of figure $11 \mathrm{~b}$ is further to suppress those features of the pulse pattern that are subject to more than inverse-square-law divergence.

Ionic attenuation also has some effect on the elliptical polarization properties of the magnetoionic waves. In particular the interpretation of (67) is affected. The projections of the electric ellipses for the two magnetoionic waves on a plane perpendicular to the direction of phase propagation, instead of being perpendicularly located, are turned through equal angles in opposite directions. Also, the condition for equality of the refractive indices of the two magnetoionic waves, instead of occurring for the hydromagnetic approximation when the direction of phase propagation is strictly transverse to the magnetic field, occurs at a small angle to this direction equal to $\nu_{i} \omega_{M i} / \omega_{N i}^{2}$.

\section{References}

Alfvén, H. (1950), Cosmical electrodynamics (Oxford University Press, Oxford, Eng.).

Allis, W. P. (1959), MIT Research Laboratory of Electronics Quarterly Progress Report 54, 5.

Allis, W. P., S. J. Buchsbaum, and A. Bers (1963), Waves in anisotropic plasmas, (MIT Press).

Appleton, E. V. (1932), Wireless studies of the ionosphere, J. Inst. Elect. Engrs. 71, 642-650.

Aström, E. (1950), On waves in an ionized gas, Arkiv. Fysik 2, No. 42, 443-457.

Auer, P. L., H. Hurwitz, Jr., and R. D. Miller (1958), Collective oscillations in a cold plasma, Phys. Fluids 1, No. 6, 501-514.

Bershader, D. (editor) (1959), The magnetodynamics of conducting fluids (Stanford University Press).

Booker, H. G. (1934), Some general properties of the formulae of the magneto-ionic theory, Proc. Roy. Soc. (London) 147A, $352-382$.

Booker, H. G. (1935), The application of the magneto-ionic theory to the ionosphere, Proc. Roy. Soc. (London) 150A, 267-286.

Budden, K. G. (1961), Radio waves in the ionosphere (Cambridge University Press).

Clemmow, P. C., and R. F. Mullaly (1955), Physics of the Ionosphere, Report of Physical Society Conference, Cavendish Laboratory, 340 .

Denisse, J. F., and J. L. Delcroix (1961), Théorie des Ondes dans les Plasmas, Dunod, Paris.

Eckersley, T. L. (1935), Musical atmospherics, Nature 135, 104.

Gendrin, R. (1960), Guidage des sifflments radio electriques par le champ magnetique terrestre, Comptes Rendus, Acad. Sci. Paris 251, 1085-1087.

Hartree, D. R. (1931), The propagation of electromagnetic waves in a refracting medium in a magnetic field, Proc. Camb. Phil. Soc. 27, 143-162.

Hines, C. O. (1957), Heavy-ion effects in audio-frequency radio propagation, J. Atmospheric Terrest. Phys. $11,36-42$.

Lighthill, M. J. (1960), Studies on magneto-hydrodynamic waves and other anisotropic motions, Trans. Roy. Soc. (London) 252A, 397-430.

Ratcliffe, J. A. (1959), The magneto-ionic theory and its applications to the ionosphere (Cambridge University Press). Smith, R. L. (1960), Guiding of whistlers in a homogeneous medium, J. Res. NBS 64D (Radio Prop.), No. 5, 505-508.

Stix, T. H. (1962), The theory of plasma waves (McGraw-Hill Book Co., Inc., New York, N.Y.).

(Paper 69D4-484) 